\title{
AMP-Activated Protein Kinase Regulates Circadian Rhythm by Affecting CLOCK in Drosophila
}

\author{
Eunjoo Cho, ${ }^{1,2 *}$ Miri Kwon, ${ }^{1,2 *}$ Jaewon Jung, ${ }^{1,2 *}$ Doo Hyun Kang, ${ }^{1,2}$ Sanghee Jin, ${ }^{2}$ Sung-E Choi, ${ }^{3}{ }^{-}$Y up Kang, ${ }^{3}$ \\ and ${ }^{\circ}$ Eun Young Kim $^{1,2}$ \\ ${ }^{1}$ Neuroscience Graduate Program, Department of Biomedical Sciences, Ajou University Graduate School of Medicine, ${ }^{2}$ Department of Brain Science, Ajou \\ University School of Medicine, and ${ }^{3}$ Department of Physiology, Ajou University School of Medicine, Suwon, Kyunggi-do, 16499, Republic of Korea.
}

The circadian clock organizes the physiology and behavior of organisms to their daily environmental rhythms. The central circadian timekeeping mechanism in eukaryotic cells is the transcriptional-translational feedback loop (TTFL). In the Drosophila TTFL, the transcription factors CLOCK (CLK) and CYCLE (CYC) play crucial roles in activating expression of core clock genes and clock-controlled genes. Many signaling pathways converge on the CLK/CYC complex and regulate its activity to fine-tune the cellular oscillator to environmental time cues. We aimed to identify factors that regulate CLK by performing tandem affinity purification combined with mass spectrometry using Drosophila S2 cells that stably express HA/FLAG-tagged CLK and V5-tagged CYC. We identified SNF4A $\gamma$, a homolog of mammalian AMP-activated protein kinase $\gamma(\mathrm{AMPK} \gamma)$, as a factor that copurified with HA/FLAG-tagged CLK. The AMPK holoenzyme composed of a catalytic subunit AMPK $\alpha$ and two regulatory subunits, AMPK $\beta$ and AMPK $\gamma$, directly phosphorylated purified CLK in vitro. Locomotor behavior analysis in Drosophila revealed that knockdown of each AMPK subunit in pacemaker neurons induced arrhythmicity and long periods. Knockdown of AMPK $\beta$ reduced CLK levels in pacemaker neurons, and thereby reduced pre-mRNA and protein levels of CLK downstream core clock genes, such as period and vrille. Finally, overexpression of CLK reversed the long-period phenotype that resulted from AMPK $\beta$ knockdown. Thus, we conclude that AMPK, a central regulator of cellular energy metabolism, regulates the Drosophila circadian clock by stabilizing CLK and activating CLK/CYC-dependent transcription.

Key words: AMP-activated protein kinase; circadian clock; CLOCK; Drosophila; tandem affinity purification; TTFL

Significance Statement

Regulation of the circadian transcription factors CLK and CYC is fundamental to synchronize the core clock with environmental changes. Here, we show that the AMPK $\gamma$ subunit of AMPK, a central regulator of cellular metabolism, copurifies with the CLK/CYC complex in Drosophila S2 cells. Furthermore, the AMPK holoenzyme directly phosphorylates CLK in vitro. This study demonstrates that AMPK activity regulates the core clock in Drosophila by activating CLK, which enhances circadian transcription. In mammals, AMPK affects the core clock by downregulating circadian repressor proteins. It is intriguing to note that AMPK activity is required for core clock regulation through circadian transcription enhancement, whereas the target of AMPK action is different in Drosophila and mammals (positive vs negative element, respectively).

\section{Introduction}

The circadian clock allows living organisms to manifest daily rhythms in physiology and behavior by synchronizing internal

Received Sept. 11, 2018; revised Feb. 19, 2019; accepted Feb. 22, 2019.

Author contributions: E.C. and E.Y.K. designed research; E.C., M.K., J.J., D.H.K., and S.J. performed research; E.C., M.K., J.J., and E.Y.K. analyzed data; S.-E.C. and Y.K. contributed unpublished reagents/analytic tools; E.Y.K. wrote the paper.

This work was supported by the National Research Foundation of Korea, funded by the Korea government MSIP 2017R1A2B2010334 and 2012R1A5A048183. We thank Paul E. Hardin (Texas A\&M) for generously providing antiCLK and anti-VRI antibodies; Thomas Kusch (Rutgers University) for providing the pMT-HA/FLAG plasmid; and Joungkyeong Chung (Seoul National University) for providing the AMPK null mutants.

The authors declare no competing financial interests.

*E.C., M.K., and J.J. contributed equally to this work.

Correspondence should be addressed to Eun Young Kim at ekim@ajou.ac.kr. processes with rhythmic environmental changes. In eukaryotes, the cell-autonomous circadian clock operates via transcriptionaltranslational feedback loops (TTFLs) composed of core clock proteins. In Drosophila, CLOCK (CLK) and CYCLE (CYC) activate the transcription of period (per) and timeless (tim), and PER and TIM proteins, in turn, repress their own transcription in the core loop. CLK and CYC also activate the transcription of vrille (vri) and the PAR domain protein $1 \varepsilon$ gene $(P d p 1 \varepsilon)$. VRI and $\mathrm{PDP} 1 \varepsilon$ repress and activate the transcription of $C l k$, which enhances the robustness of the interlocked feedback loop. CLOCK- 
WORK ORANGE (CWO), another downstream target of CLK/ CYC, works cooperatively with PER to increase the amplitude of oscillation (Hardin, 2011). A similar mechanism operates in mammals with CLK/BMAL1 as the master circadian transcription factors and PER1, PER2, and PER3, and CRYPTOCHROME1 and 2 (CRY1, CRY2) as the transcriptional repressors in the core loop. Nuclear receptors RORa and REB-erb $\alpha$, which are induced by CLK/BMAL1, activate and inhibit the expression of BMAL1, respectively, to form an interlocked feedback loop (Partch et al., 2014).

In the Drosophila melanogaster brain, $\sim 150$ pacemaker neurons strongly express the core clock genes and drive the locomotor activity rhythms (Helfrich-Förster, 2005). The pacemaker neurons form clusters, and each cluster performs a specific function that determines activity profiles in response to the environmental inputs. The dorsal neurons are $\mathrm{DN}_{\mathrm{a}} \mathrm{s}, \mathrm{DN1} \mathrm{p}_{\mathrm{p}} \mathrm{s}, \mathrm{DN} 2 \mathrm{~s}$, and $\mathrm{DN} 3$ s. The lateral clusters are the ventrally located neurons $\left(\mathrm{LN}_{\mathrm{v}} \mathrm{s}\right)$, including the large lateral ventral neurons $\left(\mathrm{LN}_{\mathrm{v}} \mathrm{s}\right)$, the small lateral ventral neurons $\left(\mathrm{sLN}_{\mathrm{v}} \mathrm{s}\right)$, the fifth $\mathrm{LN}_{\mathrm{v}}$, and the lateral dorsal neurons $\left(\mathrm{LN}_{\mathrm{d}} \mathrm{s}\right)$. Among the pacemaker neurons, the $s L N_{v} s$ are crucial for rhythmic behavior under constant conditions (Helfrich-Förster, 1998; Renn et al., 1999; Nitabach et al., 2002). Further, $s_{L N}$ s project dorsally and terminate in the vicinity of DN1s and DN2s and express peptide dispersing factor (PDF), a neuropeptide that allows $\mathrm{sLN}_{\mathrm{v}} \mathrm{s}$ to coordinate pacemaker neurons to maintain synchrony (Renn et al., 1999; Lin et al., 2004; Helfrich-Förster et al., 2007)

Because CLK is the rate-limiting component of TTFL, maintaining the appropriate CLK activity and level is crucial for circadian rhythm generation. Increasing the transcription activation potency of CLK increases the speed of the circadian clock (Kadener et al., 2008; Lee et al., 2014), and flies harboring the hypomorphic allele $C l k^{\text {ar }}$ that has reduced CLK transcription activity do not exhibit circadian rhythms (Allada et al., 2003). The phosphorylation pattern of CLK also has daily rhythms that tightly couple with the rhythms of activity and stability. Early day and late night hyperphosphorylation is primarily driven by the $D$. melanogaster kinase DOUBLETIME (DBT), which phosphorylates PER and is the homolog of the mammalian casein kinase I $\delta / \varepsilon$, reduces CLK stability, and correlates with reduced transcriptional activity (Kim and Edery, 2006; Yu et al., 2006; Menet et al., 2010). Consistently, dephosphorylation of CLK by protein phosphatase 2A (PP2A) induces stabilization and enhances transcriptional activity (Kim and Edery, 2006; Andreazza et al., 2015). NEMO, another kinase that phosphorylates PER, has also been shown to destabilize CLK in flies (Yu et al., 2011; Szabó et al., 2013), but casein kinase 2 (CK2) is recruited onto CLK during late night but makes CLK stable and inactive (Szabó et al., 2013). During mid-day when CLK retains high transcriptional activity, CLK is present in the medium to hypophosphorylated isoforms. This mid-day specific phosphorylation occurs on the $16 \mathrm{Ser} / \mathrm{Thr}$ residues by yet unidentified kinases (Lee et al., 2014) and maintains the appropriate levels and transcriptional activity of CLK, which is crucial to synchronize animal behavior to the temperature cycle.

In this study, we aimed to isolate proteins that interact with CLK/CYC to understand how CLK/CYC activity is regulated. $D$. melanogaster S2 cells that stably expressed FLAG/HA epitopetagged CLK and V5 epitope-tagged CYC were used for tandem affinity purification (TAP) followed by mass spectrometry (MS) analysis. We identified SNF4A $\gamma$, the regulatory subunit of the AMP-activated protein kinase (AMPK) holoenzyme, as a protein that interacts with CLK/CYC. Downregulation of each subunit of the AMPK holoenzyme in pacemaker cells significantly altered circadian locomotor behavior, indicating that the enzymatic activity of AMPK is required for normal circadian clock function. Moreover, the AMPK holoenzyme directly phosphorylated GST-tagged CLK proteins in vitro. Because CLK protein levels were reduced in clock cells and because the ectopic expression of CLK rescued the period-lengthening defect of the AMPK $\beta$ subunit knockdown, we concluded that the major cellular energy sensor AMPK affects the core clock by stabilizing CLK in D. melanogaster.

\section{Materials and Methods}

Plasmids. To generate the pMT-Clk-HA/FLAG plasmid, the Clk open reading frame was amplified from the $\mathrm{SK}(-) / C l k$ plasmid (Lee et al., 1999) and cloned into a pMT-HA/FLAG expression vector carrying a hygromycin resistance cassette (Kusch et al., 2004). The SNF4A $\gamma$ open reading frame was PCR-amplified from $\mathrm{CDNA}$ that had been reversetranscribed in a manner that excluded the stop codon from total RNA extracted from fly heads, and the PCR product was subcloned into the pMT-V5-His vector (Invitrogen). The PCR primers used were $5^{\prime}$ ATTGGTACCATGCATGGAATAACACACCT-3' and 5'-ATTTCTAGA TTFACTAACCAACGCCATTT-3'; the SNF4A $\gamma$ sequences are underlined. The pMT- $c y c$-V5 and pMT-HA-Clk plasmids were described previously (Kim and Edery, 2006).

TAP. D. melanogaster S2 cells were cotransfected with $9.5 \mu \mathrm{g}$ each of the pMT-Clk-HA/FLAG and pMT-cyc-V5 plasmids using calcium phosphate (Invitrogen) according to the manufacturer's protocol. Stable transgenic S2 cell lines harboring the pMT-Clk-HA/FLAG and pMT$c y c-\mathrm{V} 5$ plasmids were selected by culturing in a hygromycin-containing medium. Stable transgenic S2 cells were expanded in a $2 \mathrm{~L}$ suspension culture, and CLK-HA/FLAG and CYC-V5 expression was induced by the addition of $\mathrm{CuSO}_{4}$ to a final concentration of $500 \mu \mathrm{M}$. After a $1 \mathrm{~d}$ induction, the cells were harvested, and proteins were extracted in lysis buffer [50 mм Tris- $\mathrm{HCl}, \mathrm{pH}$ 7.5, $150 \mathrm{~mm} \mathrm{NaCl}, 0.4 \%$ (v/v) NP-40, $1.5 \mathrm{~mm}$ $\mathrm{MgCl}_{2}, 5 \%$ glycerol, 1 mм EDTA, 1 mм DTT, 1 mм Na $\mathrm{VO}_{4}, 2.5 \mathrm{~mm} \mathrm{NaF}$, and a freshly added protease inhibitor mixture (Sigma-Aldrich)]. Protein extracts were incubated with $0.6 \mathrm{ml}$ of EZview Red anti-FLAG M2 affinity gel (Sigma-Aldrich, RRID:AB_2616449) overnight at $4^{\circ} \mathrm{C}$ with rotation. The gel was then washed three times with lysis buffer and eluted using the $3 \times$ FLAG peptide $(1.5 \mathrm{mg} / \mathrm{ml})$. The eluate was diluted with lysis buffer and incubated with EZview Red anti-HA affinity gel (Sigma-Aldrich, RRID:AB_10109562) for $3 \mathrm{~h}$ at $4^{\circ} \mathrm{C}$ with rotation. The gel was then washed three times with lysis buffer and eluted using the $2 \mathrm{HA}$ peptide $(1 \mathrm{mg} / \mathrm{ml})$. The final eluate was used for MS analysis (DiatechKorea)

Nano-liquid chromatography (LC)-MS/MS analysis. Proteins in the eluate were separated by SDS-PAGE and stained with Coomassie Brilliant Blue. Protein bands were excised from the gel, and the gel pieces were vortexed in $50 \%$ acetonitrile (ACN) containing $50 \mathrm{~mm} \mathrm{NH}_{4} \mathrm{HCO}_{3}$ until the stain was completely removed. The gel pieces were then dehydrated in $100 \%$ ACN and vacuum-dried for 20 min using a SpeedVac. For in-gel digestion, the disulfide bridges were reduced with $10 \mathrm{~mm}$ DTT in $50 \mathrm{~mm}$ $\mathrm{NH}_{4} \mathrm{HCO}_{3}$ for $45 \mathrm{~min}$ at $56^{\circ} \mathrm{C}$, and then the cysteines were alkylated with $55 \mathrm{~mm}$ iodoacetamide in $50 \mathrm{mM} \mathrm{NH}_{4} \mathrm{HCO}_{3}$ for $30 \mathrm{~min}$ in the dark. Finally, each gel piece was treated with $12.5 \mathrm{ng} / \mu \mathrm{l}$ sequencing-grade modified trypsin (Promega) in $50 \mathrm{~mm} \mathrm{NH}_{4} \mathrm{HCO}_{3}$ buffer, $\mathrm{pH} 7.8$, at $37^{\circ} \mathrm{C}$ overnight. Following digestion, tryptic peptides were extracted with $5 \%$ formic acid in $50 \% \mathrm{ACN}$ at room temperature for $20 \mathrm{~min}$. The supernatants were collected and dried using a SpeedVac. The samples were resuspended in $0.1 \%$ formic acid and purified and concentrated using C18 ZipTips (Millipore) before MS analysis. The samples were loaded onto a fused silica microcapillary column $(12 \mathrm{~cm} \times 75 \mu \mathrm{m})$ packed with $\mathrm{C} 18$ reversed-phase resin $(5 \mu \mathrm{m}, 200 \AA)$. LC was conducted using a linear gradient: a $3 \%-40 \%$ gradient of solvent $\mathrm{B}(0.1 \%$ formic acid in $\mathrm{ACN})$ in solvent A $(0.1 \%$ formic acid in distilled water) with a flow rate of 250 $\mathrm{nl} / \mathrm{min}$ for $60 \mathrm{~min}$. The column was directly connected to an LTQ linear ion-trap MS (ThermoFinnigan) equipped with a nano-electrospray ion source. Each full MS scan was followed by five MS/MS scans of the most intense to the fifth-most intense peaks of the full MS scan. The repeat 
count of peaks for dynamic exclusion was 1 , with a $30 \mathrm{~s}$ repeat duration. The dynamic exclusion duration was set to $180 \mathrm{~s}$, and the width of exclusion mass was $\pm 1.5 \mathrm{Da}$. The list size of dynamic exclusion was 50 . The acquired LC-ESI-MS/MS fragment spectra were searched for in the BioWorksBrowser (version Rev. 3.3.1 SP1, Thermo Fisher Scientific, RRID: SCR_014594) with the SEQUEST search engine against the $D$. melanogaster database at National Center for Biotechnology Information (http://www.ncbi.nlm.nih.gov/). The following search conditions were used: trypsin enzyme, up to two missed cleavages permitted, peptide tolerance of $\pm 2 \mathrm{amu}$, a mass error of $\pm 1 \mathrm{amu}$ on the fragment ions, and fixed modifications of carbamidomethylation of cysteines $(57 \mathrm{Da})$ and oxidation of methionines (16 Da).

Fly strains. The following fly strains were used: $w^{1118}$ (BDSC, BL5905), $c y c^{01}$ (Rutila et al., 1998), $C l k^{\text {out }}$ (Lee et al., 2014), $C l k^{\text {jrk }}$ (Allada et al., 1998), dcr2;tim(UAS)-Gal4 (Kim et al., 2012), UAS-Clk (Zhao et al., 2003), pdf-Gal4 (Park et al., 2000), tub-Gal80 ${ }^{\text {ts }}$;im(UAS)-Gal4 (Kim et al., 2012), UAS-AMPK $\alpha$ Ri-1 (VDRC1827), UAS-AMPK $\alpha$ Ri-2 (NIG3051R-1), UAS-AMPK $\beta$ Ri-1 (NIG8057R-2), UAS-AMPK $\beta$ Ri-2 (NIG8057R-4), UAS-AMPK $\gamma$ Ri-1 (NIG17299R-3), UAS-AMPK $\gamma$ Ri2 (NIG17299R-1), UAS-GFP.nls (BDSC, BL4776), and UAS-mCD8:: GFP (BDSC, BL5130). pdf-Gal4 was crossed to UAS-dicer2/FM6 to generate dcr2;pdf-Gal4. For AMPK subunit knockdown, driver flies [e.g., dcr2;actin-Gal4, dcr2;tim(UAS)-Gal4, dcr2;pdf-Gal4, and tub-Gal80 ${ }^{\text {ts }}$; tim(UAS)-Gal4] were crossed to UAS-AMPK RNAi flies. UAS-RNAi flies used in this study were in the $w^{1118}$ genetic background. Driver flies were crossed to $w^{1118}$ flies and served as experimental heterozygous controls (marked as actin $>d 2$, tim $>d 2$, pdf $>d 2$, and tim, G80 ${ }^{\text {ts }}>d 2$, respectively). None of the fly strains was backcrossed to $w^{1118}$ flies.

Behavioral analysis. The locomotor activity of individual flies was determined using the Drosophila Activity Monitoring system (Trikinetics). Young male adult flies were exposed to $12 \mathrm{~h}$ of light and $12 \mathrm{~h}$ of dark (LD) for $4 \mathrm{~d}$ and then were maintained in constant darkness (DD) for $7 \mathrm{~d}$ at the indicated temperatures. The locomotor data analysis was performed using FaasX software (Fly Activity Analysis Suite for MacOSX), which was generously provided by Francois Rouyer (Centre National de la Recherche Scientifique, France). Periods were calculated for each fly using $\chi^{2}$ periodogram analysis, and data were pooled to obtain an average value. Power was calculated by quantifying the relative strength of the rhythm during DD. Individual flies with a power $\geq 10$ and a width $\geq 2$ were considered rhythmic. Actograms represent the double-plotted locomotor activities throughout the experimental period and were acquired using Actogram J software (Schmid et al., 2011).

$q R T-P C R$. qRT-PCR was performed as described previously (Lee et al., 2016). Total RNA was extracted from fly heads using QIAzol reagent (QIAGEN). Total RNA $(1 \mu \mathrm{g})$ was reverse-transcribed using an oligo(dT) 20 primer (for mRNA) or a random hexamer primer (for premRNA) and PrimeScript RTase (TaKaRa). Real-time PCR was performed using Rotor Gene 6000 (QIAGEN) with SYBR Premix Ex Taq (Tli RNaseH Plus, TaKaRa). The following primers were used: $a m p k \alpha$ forward, 5' -CCGGCAAGTTCTCGAAGAT-3'; ampk $\alpha$ reverse, 5' -GAG TAAGGCTCTCCATGATGAC-3'; ampk $\beta$ forward, 5'-GTGACCAAC TATGCGGAGAA-3'; ampk $\beta$ reverse, 5' -AGCGGTGTATCCTTGTTG AG-3'; ampk $\gamma$ forward, 5' -ATCGAGGACATACCCGAAGA-3'; ampk $\gamma$ reverse, 5'-GTCTGGTCGTGCTGGTTATT-3'; per forward, 5'-GT GAGAGCGAGAGCGAGTGT-3'; per reverse, 5'-TATGTAAGCTGC CTGCCCAA-3'; vri forward, 5' -CAAGCAGGATAATCCCAGCAA-3'; and vri reverse, 5'-ACAGATTTCAAGATCAAACGTGGA-3'. Noncycling mRNA encoding $c b p 20$ was used to normalize gene expression with the following primers: $c b p 20$ forward, $5^{\prime}$-GTATAAGAAGACGCCCTGC-3'; and $c b p 20$ reverse, 5'-TTCACAAATCTCATGGCCG-3'. The data were analyzed using Rotor Gene 6000 software, and the relative mRNA levels were quantified using the $2^{-\Delta \Delta \mathrm{Ct}}$ method in which $\Delta \Delta \mathrm{Ct}=$ $\left[\left(\mathrm{C}_{\mathrm{t}}\right.\right.$ target $\left.-\mathrm{C}_{\mathrm{t}} \operatorname{cbp} 20\right)$ at ZTx $]-\left[\left(\mathrm{C}_{\mathrm{t}}\right.\right.$ target $\left.-\mathrm{C}_{\mathrm{t}} \operatorname{cbp} 20\right)$ at ZT4 $]$.

Western blotting and immunoprecipitation. For Western blotting, protein extracts were prepared in HEMG lysis buffer [ 10 mM HEPES, pH 7.5, 5 mм Tris- $\mathrm{HCl}$, pH 7.5, $50 \mathrm{~mm} \mathrm{KCl,} \mathrm{10 \%} \mathrm{glycerol,} \mathrm{0.1 \%} \mathrm{Triton} \mathrm{X-100,} 1$ mM EDTA, and $1 \mathrm{~mm}$ DTT] with freshly added protease inhibitor mixture (Sigma-Aldrich), $1 \mathrm{~mm} \mathrm{Na} \mathrm{VO}_{4}$, and $1 \mathrm{~mm} \mathrm{NaF}$. To detect dCLK, protein extracts were prepared in RIPA lysis buffer $[25 \mathrm{~mm}$ Tris- $\mathrm{HCl}, \mathrm{pH}$
7.5, $50 \mathrm{~mm} \mathrm{NaCl}, 0.5 \%$ sodium deoxycholate, $0.5 \% \mathrm{NP}-40$, and $0.1 \%$ SDS] and were briefly sonicated (Kim and Edery, 2006). Protein extracts were resolved by SDS-PAGE with the indicated polyacrylamide percentage or on $3 \%-8 \%$ Tris-acetate Criterion gels (for CLK). Primary antibodies were used at the following dilutions: anti-CLK (gp208), 1:2000; anti-V5 (Invitrogen, RRID:AB_159298), 1:2000; anti-HA (Roche Diagnostics, RRID:AB_514505), 1:2000; anti-PER (Rb1), 1:2000; anti-TIM (Rb1), 1:2000; and anti-AMPK $\alpha$ (AbD Serotec, RRID:AB_1604624), 1:2000. Band intensity was quantified using ImageJ software (RRID:SCR_003070).

For immunoprecipitation, protein extracts were prepared in modified-RIPA lysis buffer (50 mм Tris-HCl, pH 7.5, 150 mм NaCl, 1\% NP-40, and $0.25 \%$ sodium deoxycholate) and were briefly sonicated. Then, $3 \mu$ l of anti-V5 (Invitrogen, RRID:AB_159298) or anti-HA (Roche Diagnostics, RRID:AB_514506) was added to the extract depending on the target protein and incubated overnight at $4^{\circ} \mathrm{C}$ with end-over-end rotation. Normal rabbit IgG (Santa Cruz Biotechnology) was used as a negative control. Finally, $20 \mu \mathrm{l}$ of Gamma Bind-Sepharose beads (GE Healthcare) was added, and the mixture was further incubated for $3 \mathrm{~h}$ at $4^{\circ} \mathrm{C}$ with end-over-end rotation. The immune complexes were then eluted from the beads using SDS-PAGE sample buffer.

Immunostaining. Immunostaining was performed as previously described (Lee et al., 2016). Briefly, fly heads were fixed in 4\% PFA and washed with $0.5 \%$ Triton X-100 (PBT). The fixed heads were dissected, the isolated brains were permeabilized in $1 \%$ PBT for $20 \mathrm{~min}$, and then blocked in $0.5 \%$ PBT containing $5 \%$ horse serum for $30 \mathrm{~min}$. The following primary antibodies were added to the mixtures directly: anti-PDF antibody (C7) (Cyran et al., 2005), diluted 1:200; anti-VRI (gp2) (Glossop et al., 2003), diluted 1:1000; anti-PER (Rb1) (Kim et al., 2012), diluted 1:200; and anti-CLK (gp50) (Houl et al., 2006), diluted 1:200. The brains were washed with PBT and incubated with secondary antibodies in a blocking solution overnight at $4^{\circ} \mathrm{C}$. The following secondary antibodies were used at 1:200 dilution: goat anti-rabbit Alexa-488 (Thermo Fisher Scientific, RRID:AB_143165), goat anti-mouse Alexa-555 (Thermo Fisher Scientific, RRID:AB_141780), and goat anti-mouse Alexa-633 (Thermo Fisher Scientific, RRID:AB_2535718). Stained brain samples were washed with $\mathrm{PBT}$, incubated in $0.1 \mathrm{M}$ phosphate buffer containing $50 \%$ glycerol for $30 \mathrm{~min}$, and mounted using a mounting medium. Confocal images were obtained using an LSM 710 confocal microscope (Carl Zeiss) and processed using the Zen software (ZEN Digital Imaging for Light Microscopy, Carl Zeiss, RRID:SCR_013672). For signal quantification, the pixel intensity of each cell was determined using ImageJ software. Briefly, the level of background staining was measured in the field surrounding the cluster of clock neurons and subtracted from the pixel intensities determined for the clock neuronal cells. The final intensity was the average of at least five brains for each genotype.

In vitro kinase assay. GST-tagged recombinant CLK proteins were purified from Escherichia coli BL21 cells, which were transformed with pGEX4T-1- $d C l k$, using the MagneGST protein purification system (Promega). Briefly, E. coli pellets were lysed with lysis buffer (MagneGST cell lysis reagent, Promega) containing $1 \mathrm{mg} / \mathrm{ml}$ lysozyme (Sigma-Aldrich), RNase-free DNase, and $5 \mathrm{~mm}$ DTT added fresh. MagneGST particles were equilibrated with lysis buffer and incubated with the lysates for $3 \mathrm{~h}$ at $4^{\circ} \mathrm{C}$ with rotation. Magnetic particles with bound GST-tagged CLK were collected using a magnetic stand and were then washed with lysis buffer three times. Protein was eluted with a $50 \mathrm{~mm}$ Tris-HCl, $\mathrm{pH}$ 8.1, buffer containing $50 \mathrm{~mm}$ glutathione.

The in vitro kinase assay was performed with $1 \mu \mathrm{g}$ of purified GSTCLK as the substrate and $50 \mathrm{ng}$ active AMPK holoenzyme (Millipore) in $20 \mathrm{~mm}$ HEPES, pH 7.5, $10 \mathrm{~mm} \mathrm{MgCl}_{2}, 1 \mathrm{~mm}$ DTT, $6.25 \mathrm{~mm}$ $\beta$-glycerophosphate, $1.25 \mathrm{~mm}$ EGTA, and $0.125 \mathrm{~mm}$ cold ATP (or with 12 $\mu \mathrm{Ci} \gamma-{ }^{32} \mathrm{P}$-ATP for the radioactive assay) in the presence or absence of $0.15 \mathrm{~mm}$ AMP at $30^{\circ} \mathrm{C}$ for $20 \mathrm{~min}$. The samples were resolved by SDSPAGE and then transferred to a PVDF membrane. The membrane was stained with Ponceau S, and phosphorylation of the substrate was visualized by autoradiography.

Statistical analysis. Statistical analysis was performed using Prism version 7.0 software (GraphPad; RRID:SCR_002798). Significant differences between groups were determined using the Student's $t$ test, one- 
A

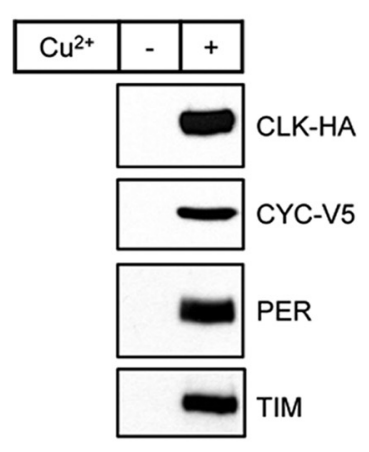

B

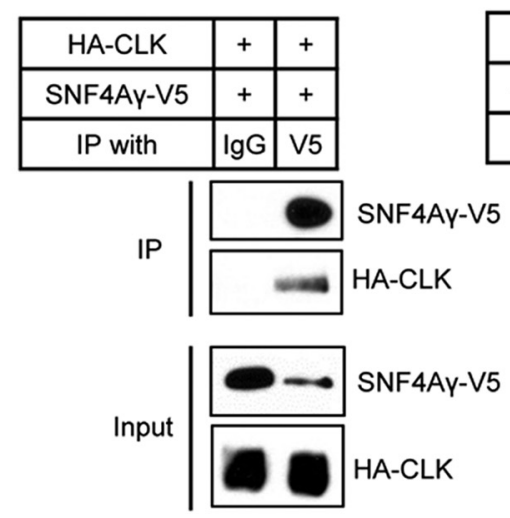

C

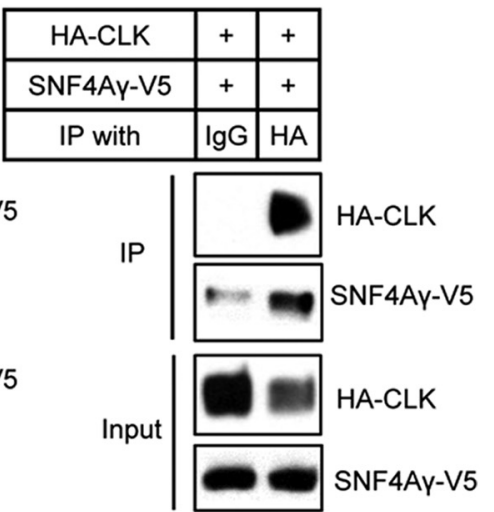

Figure 1. Confirmation of the interaction between AMPK $\gamma$ and CLK by reciprocal coimmunoprecipitation. $A$, S2 cells that stably expressed TAP-tagged CLK and V5-tagged CYC were treated with $(+)$ or without (-) $500 \mu \mathrm{M} \mathrm{CuSO}{ }_{4}$ (final concentration). Then, $24 \mathrm{~h}$ after induction, protein extracts were analyzed by immunoblotting. The expression of TAP-tagged CLK and V5-tagged CYC induced expression of endogenous PER and TIM proteins as shown on Western blots probed with anti-PER antibody (Rb1) and anti-TIM antibody (Rb1). $\boldsymbol{B}, \boldsymbol{C}$, S2 cells were transiently transfected with pMT-HA-Clk and pMT-SNF4A $\gamma$-V5, and expression of HA-CLK and AMPK $\gamma$-V5 was induced by the addition of $500 \mu \mathrm{m} \mathrm{CuSO}_{4}$. Then, $24 \mathrm{~h}$ after induction, protein extracts were analyzed directly by immunoblotting (Input) or subjected to immunoprecipitation (IP) with anti-HA antibody, anti-V5 antibody, or anti-normal lgG antibody as a negative control.

way ANOVA, or two-way ANOVA followed by the Tukey's test or the Dunnett's test as indicated in the figure legends.

\section{Results \\ TAP analysis identified AMPK $\gamma$ as a protein that interacts with CLK}

To identify proteins that interact with CLK, we performed TAP with extracts from S2 cells that stably expressed HA/FLAG tandemly tagged CLK (TAP-tagged CLK) and V5-tagged CYC. We reasoned that because most CLK, if not all, functions as a heterodimer with CYC, coexpression of CYC with CLK would increase the formation of functionally intact CLK-CYC complexes. To determine whether CLK/CYC activity was affected by the tags, PER and TIM protein levels were measured in stable S2 cells. As reported previously (Saez and Young, 1996), the PER and TIM proteins were not detectable in stable S2 cells without the induction of CLK and CYC (Fig. 1A). However, $24 \mathrm{~h}$ after induction, PER and TIM protein expression was enhanced and detectable, indicating that the epitope-tagged CLK and CYC were functional and able to activate transcription (Fig. $1 A$ ).

We next purified TAP-tagged CLK and the associated proteins from total protein lysates of S2 cells, and identified the associated proteins by LC-MS/MS. In addition to CLK and CYC, the core clock proteins, including TIM, PER, DBT, and Supernumerary Limb, were among the top-hit identified proteins (data not shown), indicating that TAP successfully recovered the functional CLK/CYC complex. Among the associated proteins that were identified, we focused on SNF4A $\gamma$, which encodes the $\gamma$ regulatory subunit of AMPK and is an ortholog of mammalian Prkag1, Prkag2, and Prkag3. SNF4A $\gamma$ is henceforth referred to as AMPK $\gamma$ (Pan and Hardie, 2002). The interaction between CLK and AMPK $\gamma$ was verified by immunoprecipitation; ectopically expressed HA-tagged CLK and V5-tagged AMPK $\gamma$ were coimmunoprecipitated from S2 cells in a reciprocal manner (Fig. $1 B, C)$.

\section{Knockdown of expression of each AMPK subunit altered circadian locomotor rhythms in Drosophila}

AMPK functions as a holoenzyme consisting of a catalytic subunit ( $\alpha$ subunit) and two regulatory subunits ( $\beta$ and $\gamma$ subunits). In mammals, the $\alpha$ subunit is encoded by two isoforms, the $\beta$ subunit is encoded by two isoforms, and the $\gamma$ subunit is encoded by three isoforms. In contrast, in Drosophila, each subunit is encoded by one isoform; snflA encodes the $\alpha$ subunit, alicorn encodes the $\beta$ subunit, and snf $4 A \gamma$ encodes the $\gamma$ subunit (Pan and Hardie, 2002). To examine the in vivo relevance of the interaction between the AMPK $\gamma$ subunit and CLK, we analyzed the locomotor behavior of flies in which expression of each AMPK subunit was downregulated in timeless-expressing cells using a binary GAL4/UAS system (Brand and Perrimon, 1993) To enhance the RNA interference efficiencies, we coexpressed the UAS-dicer2 (UAS-d2) transgene. The circadian locomotor rhythms of knockdown flies were compared with two lines of control flies, timGal4; $d 2$ crossed to $w^{1118}$ (tim $>d 2$ ) and UAS-AMPK ${ }^{\text {Ri }}$ (Table 1). During the LD cycle, control flies exhibited a typical bimodal locomotor activity at times that demonstrated anticipation of the light-on and light-off periods (Fig. $2 A$, top), which subsequently persisted under DD (Fig. $2 A$, bottom). Control flies exhibited 23.7-24.5 heriod with $30 \%-98 \%$ rhythmicity depending on the lines. In $\mathrm{AMPK} \alpha$ knockdown $\left(\operatorname{tim}>d 2, \mathrm{AMPK} \alpha^{\mathrm{Ri}}-1\right.$ and tim $>d 2$, AMPK $\alpha^{\text {Ri }}-2$, named AMPK $\alpha$ Ri-1 and AMPK $\alpha$ Ri-2, respectively) and $A M P K \beta$ knockdown $\left(\operatorname{tim}>d 2, A M P K \beta^{\mathrm{Ri}}-1\right.$ and $\operatorname{tim}>d 2, A M P K \beta^{\mathrm{Ri}}-2$, named AMPK $\beta$ Ri- 1 and AMPK $\beta$ Ri-2, respectively) flies, the morning peak was similar to the peak in the control flies during the LD cycle, but the phase of the evening locomotor peak was slightly delayed (Fig. 2B, top). Consistent with a long periodicity, both morning and evening locomotor activities were delayed under DD (Fig. 2B, bottom). The phase of evening locomotor activities was slightly advanced in AMPK $\gamma$ knockdown (tim $>d 2, A M P K \gamma^{\mathrm{Ri}}-1$ and $\operatorname{tim}>d 2$, AMPK $\gamma^{\text {Ri }}-2$, named AMPK $\gamma$ Ri-1 and AMPK $\gamma$ Ri-2, respectively) flies under the LD cycle (Fig. 2B, top), and bimodal locomotor activity was absent under DD (Fig. $2 B$, bottom). AMPK $\alpha$ Ri-1 and AMPK $\alpha$ Ri-2 flies exhibited a $\sim 1$.5-h-long period with significantly reduced rhythmicity compared with control flies. AMPK $\beta$ Ri-1 flies exhibited a 1-h-long period with strong rhythmicity, and the AMPK $\beta$ Ri-2 flies exhibited a 2.5-h-long period with slightly reduced rhythmicity compared with control flies. AMPK $\gamma$ Ri-1 and AMPK $\gamma$ Ri-2 flies were arrhythmic, indicating that knockdown of AMPK $\gamma$ led to the most pronounced phenotype among the AMPK subunit knockdown flies. 
Table 1. Behavior analysis of AMPK subunit knockdown flies ${ }^{a}$

\begin{tabular}{|c|c|c|c|c|c|}
\hline Genotype & Temperature $\left({ }^{\circ} \mathrm{C}\right)$ & Number $^{b}$ & Rhythmicity (\%) & Tau & Power $^{d}$ \\
\hline tim $>$ dicer2 & 25 & 32 & 93.8 & $24.3 \pm 0.07$ & $106.7 \pm 7.84$ \\
\hline UAS-AMPK $\alpha^{\mathrm{Ri}}-1^{e}$ & 25 & 47 & 91.5 & $23.7 \pm 0.05$ & $144 \pm 9.37$ \\
\hline $\operatorname{tim}>$ dicer2, AMPK $\alpha^{\mathrm{Ri}}-1$ & 25 & 31 & 45.2 & $25.9 \pm 0.2^{k, l}$ & $42.2 \pm 5.45$ \\
\hline tim $>$ dicer2, AMPK $\alpha^{\mathrm{Ri}}-2$ & 25 & 32 & 9.7 & $25.5 \pm 0.23^{k, l}$ & $75.3 \pm 14.27$ \\
\hline UAS-AMPK $\beta^{\text {Ri }}-1^{g}$ & 25 & 48 & 75 & $24.2 \pm 0.07$ & $147.6 \pm 12.96$ \\
\hline tim $>$ dicer2, $\mathrm{AMPKK} \beta^{\mathrm{Ri}}-1$ & 25 & 32 & 90.6 & $25.3 \pm 0.45$ & $161 \pm 14.38$ \\
\hline UAS-AMPK $\gamma^{\mathrm{Ri}}-1^{i}$ & 25 & 39 & 89.7 & $24.5 \pm 0.07$ & $132 \pm 10.26$ \\
\hline $\operatorname{tim}>$ dicer2, AMPK $\gamma^{\mathrm{Ri}}-1$ & 25 & 31 & 3.2 & Arrhythmic & NA \\
\hline UAS-AMPK $\gamma^{\mathrm{Ri}}-2^{j}$ & 25 & 41 & 29.3 & $24.2 \pm 0.07$ & $90.4 \pm 14.5$ \\
\hline $\operatorname{tim}>$ dicer2, AMPK $\gamma^{\mathrm{Ri}}-2$ & 25 & 29 & 0 & Arrhythmic & NA \\
\hline tub-G80 ${ }^{\text {ts }}$, tim $>$ dicer2 & 18 & 31 & 80.6 & $23.8 \pm 0.1$ & $94.6 \pm 7.09$ \\
\hline tub-G80 ${ }^{\text {ts }}$, tim $>$ dicer 2 & 29 & 30 & 96.7 & $23.2 \pm 0.41$ & $206.2 \pm 13.32$ \\
\hline tub-G80 ${ }^{\text {ts }}$, tim $>$ dicer2, AMPK $\gamma^{\text {Ri }}-2$ & 18 & 31 & 45.2 & $24.3 \pm 0.09$ & $76.2 \pm 6.63$ \\
\hline tub-G80 ${ }^{\text {ts }}$, tim $>$ dicer2, AMPK $\gamma^{\text {Ri }}-2$ & 29 & 13 & 15.4 & $23.5 \pm 0.25^{m}$ & $50.2 \pm 4.55$ \\
\hline $\mathrm{pdf}>$ dicer2 & 25 & 30 & 93.3 & $24.5 \pm 0.09$ & $156 \pm 12.84$ \\
\hline $\mathrm{pdf}>$ dicer2, $\mathrm{AMPK} \beta^{\mathrm{Ri}}-2$ & 25 & 31 & 93.5 & $26.8 \pm 0.18^{n}$ & $127.7 \pm 8.7$ \\
\hline $\mathrm{pdf}>$ dicer2, $\mathrm{Clk}$ & 25 & 30 & 73.3 & $23.5 \pm 0.22^{n}$ & $109 \pm 10.65$ \\
\hline $\mathrm{pdf}>$ dicer2, AMPKK $\beta^{\mathrm{Ri}}-2, \mathrm{Cl} k$ & 25 & 14 & 64.3 & $24 \pm 0.74^{n}$ & $77.3 \pm 11.47$ \\
\hline
\end{tabular}

${ }^{a}$ Flies were maintained at the indicated temperatures and exposed to $4 \mathrm{~d}$ of $12 \mathrm{~h}: 12 \mathrm{~h} \mathrm{LD}$ followed by $6-8 \mathrm{~d}$ of DD.

${ }^{b}$ Total number of flies that survived until the end of the testing period.

'Percentage of flies with activity rhythms exhibiting a power value $\geq 10$ and a width value $\geq 2$.

${ }^{d}$ Relative measure of the robustness of the rhythm.

eVDRC1827.

${ }^{\prime} \mathrm{NIG} 3051 \mathrm{R}-1$.

${ }^{9} \mathrm{NIG8057R-2.}$

${ }^{h}$ NIG8057R-4.

'NIG17299R-3.

jNIG17299R-1.

kSignificantly different from the UAS-Ri control under the same conditions (one-way ANOVA, Tukey's test, $p<0.05$ ).

'Significantly different from the tim $>d c r 2$ control under the same conditions (one-way ANOVA, Tukey's test, $p<0.05$ ).

${ }^{m}$ Significantly different from the $18^{\circ} \mathrm{C}$ case in the same genetic background (Student's $t$ test, $p<0.05$ ).

${ }^{n}$ Two-way ANOVA showed significant differences in the AMPK $\beta$ knockdown $\left(F_{(1,84)}=26.23, p=1.83 \times 10^{-6}\right)$, Clk overexpression $\left(F_{(1,84)}=120.03, p=5.40 \times 10^{-18}\right)$, and interaction $\left(F_{(1,84)}=37.20, p=2.95 \times 10^{-8}\right)$.

The total daily locomotor activity counts of most knockdown flies did not exhibit significant reduction compared with parental UAS-AMPK ${ }^{\mathrm{Ri}}$ control flies, although those of AMPK $\alpha$ Ri-1 and AMPK $\beta$ Ri-1 flies were reduced (Fig. $2 C$ ). Because the reduction was observed in some, but not all, lines of knockdown flies and the extent of reduction was not severe, we reasoned that downregulation of AMPK only in timeless-expressing cells did not lead to gross locomotor defects, whereas a previous report showed that pan-neuronal AMPK $\gamma$ inactivation leads to severe locomotor defects (Nagarkar-Jaiswal et al., 2017).

Reduced expression of AMPK $\alpha, \mathrm{AMPK} \beta$, and AMPK $\gamma$ mRNA was verified in AMPK $\alpha$ Ri-2, AMPK $\beta$ Ri-2, and AMPK $\gamma$ Ri-2 flies, respectively, by real-time qRT-PCR (Fig. $2 D-F$ ). The mRNA levels of the AMPK $\alpha, \beta$, and $\gamma$ subunits did not exhibit diurnal oscillations in control flies and were significantly reduced in each knockdown fly at both ZT8 and ZT20. The AMPK $\alpha$ protein level was not reduced in whole-head extracts of AMPK $\alpha$ Ri flies, probably because gene knockdown in AMPK $\alpha$ Ri flies was limited to tim-expressing cells (data not shown). Nonetheless, reduction of AMPK $\alpha$ protein level was confirmed in AMPK $\alpha$ knockdown larvae, in which expression of $\mathrm{AMPK} \alpha^{\mathrm{Ri}}-1$ or $\mathrm{AMPK} \alpha{ }^{\mathrm{Ri}}-2$ was driven by actin-Gal4 (Fig. $2 G, H$ ).

\section{AMPK subunit downregulation affected $\operatorname{sLN}_{\mathrm{v}}$ integrity and per expression in clock neurons}

To understand the circadian rhythm defects in AMPK subunit knockdown flies, we inspected PDF neurons, which play major roles in maintaining locomotor rhythms (Helfrich-Förster, 1998; Renn et al., 1999; Nitabach et al., 2002). In AMPK $\gamma$ Ri-2 flies, which showed the most severe rhythm defects compared with AMPK $\alpha$ or AMPK $\beta$ Ri- 2 flies, PDF immunostaining at ZT2 revealed that the neurites that project dorsally from the $\mathrm{sLN}_{\mathrm{v}}$ to DNs were missing, whereas these neurites were present and produced a strong signal in control flies (Fig. 3A). The morphology of the neurites that projected dorsally from the $\mathrm{sLN}_{\mathrm{v}}$ was somewhat defective in AMPK $\alpha$ Ri-2 flies, but not in AMPK $\beta$ Ri- 2 flies (Fig. 3A). To investigate whether the absence of PDF-stained dorsally projecting neurites was a consequence of low PDF expression or loss of $\mathrm{sLN}_{\mathrm{v}}$ neurons, we observed $\mathrm{LN}_{\mathrm{v}}$ neurons directly using the reporter protein nls-GFP, which allows visualization of the soma, and mCD8::GFP, which allows visualization of neurites under the control of the pdf-Gal4 driver. Although strong GFP signals were observed in the $\mathrm{ILN}_{\mathrm{v}} \mathrm{s}$ of control and AMPK $\gamma$ Ri-2 flies, the GFP signals in the $\mathrm{sLN}_{\mathrm{v}} \mathrm{s}$ of AMPK $\gamma$ $\mathrm{Ri}-2$ flies were very weak or absent compared with the signals in 

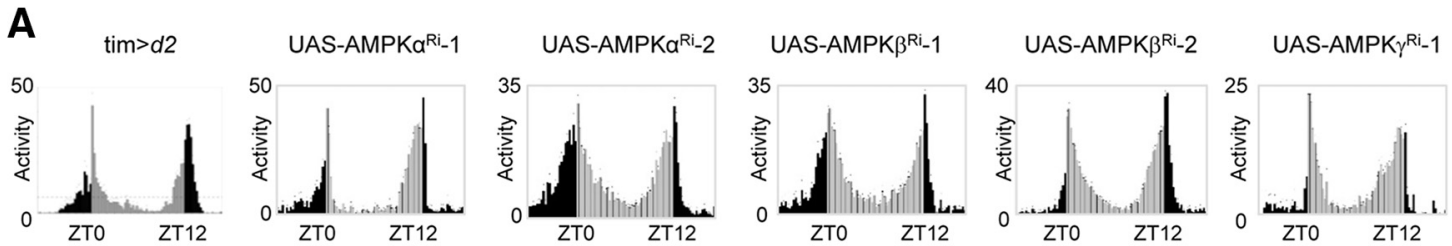

UAS-AMPK $\gamma^{R i}-2$
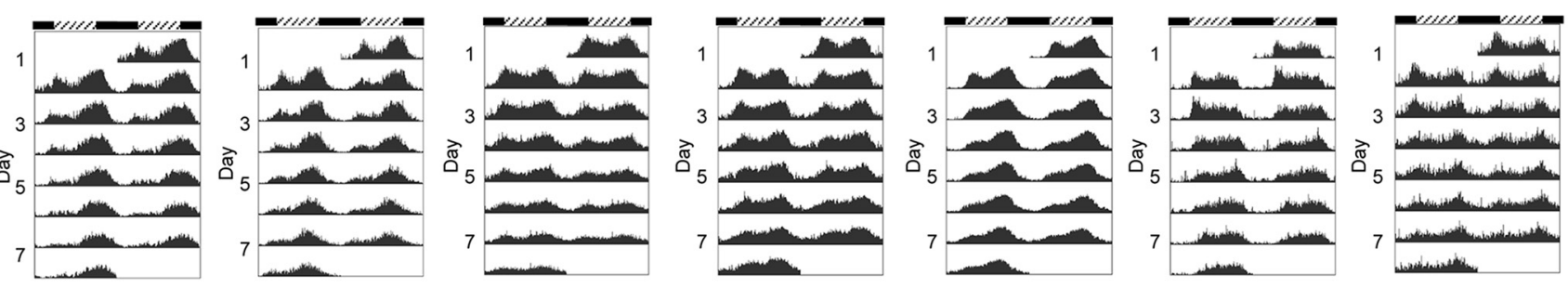

B tim>

tim>

tim>

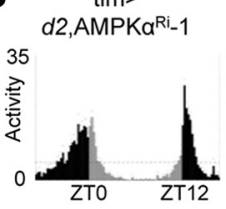

d2,AMPKa ${ }^{\text {Ri }}-2$

d2,AMPK $\beta^{\text {Ri }-1}$
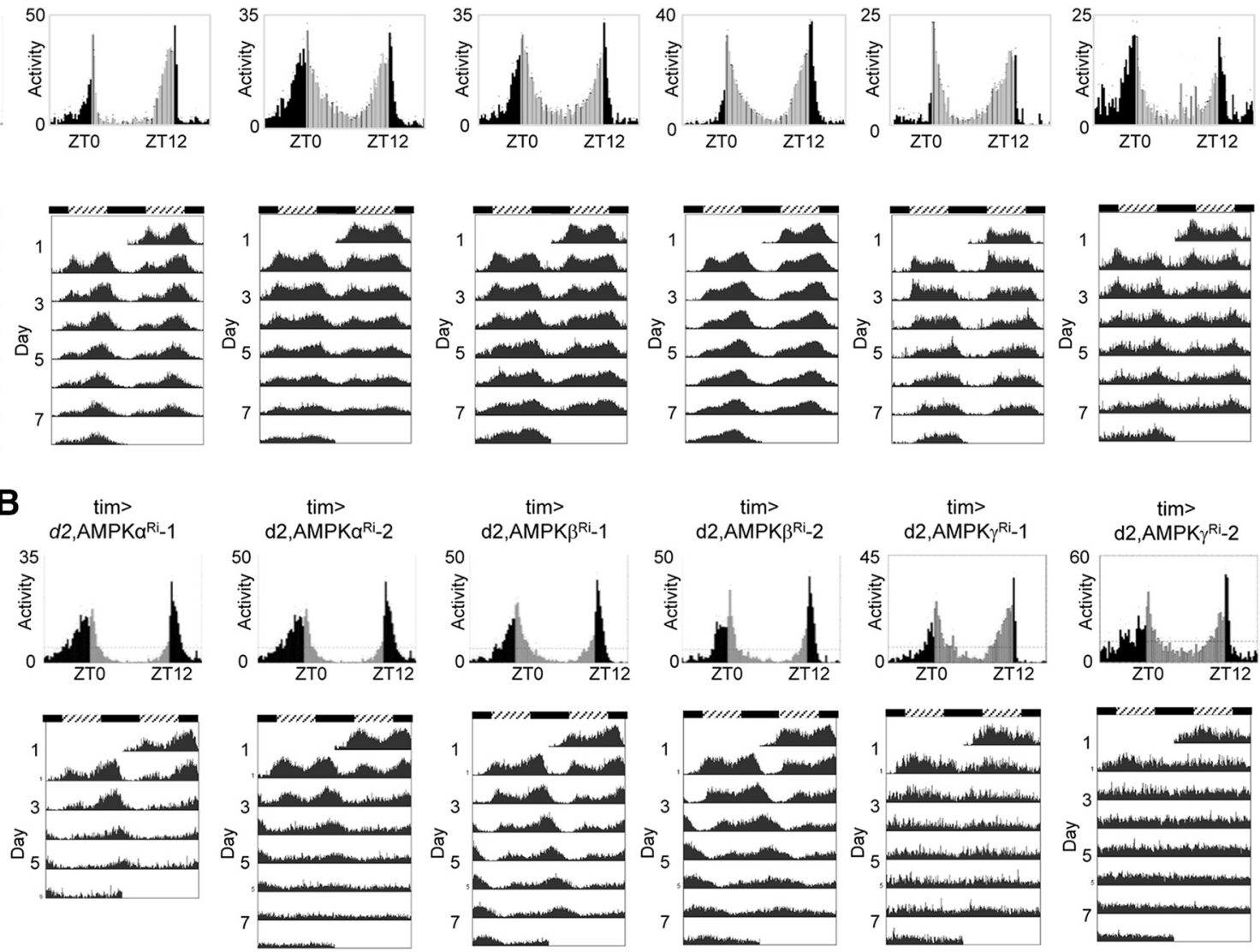

\section{C}

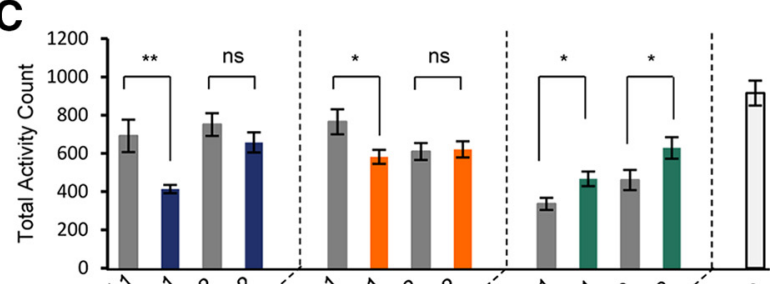

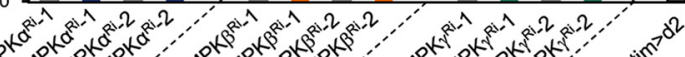
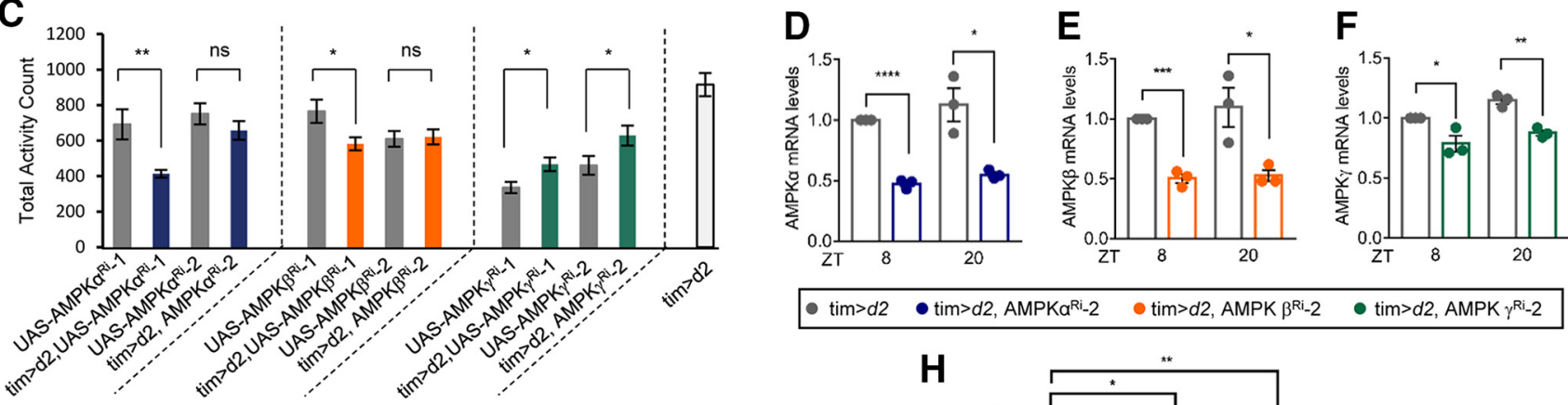

- $\operatorname{tim}>d 2 \quad \bullet$ tim $>d 2, \mathrm{AMPKa}^{\mathrm{Ri}}-2 \quad \bullet$ tim $>d 2, \mathrm{AMPK} \beta^{\mathrm{Ri}}-2$

tim>d2, AMPK $\gamma^{\mathrm{Ri}-2}$
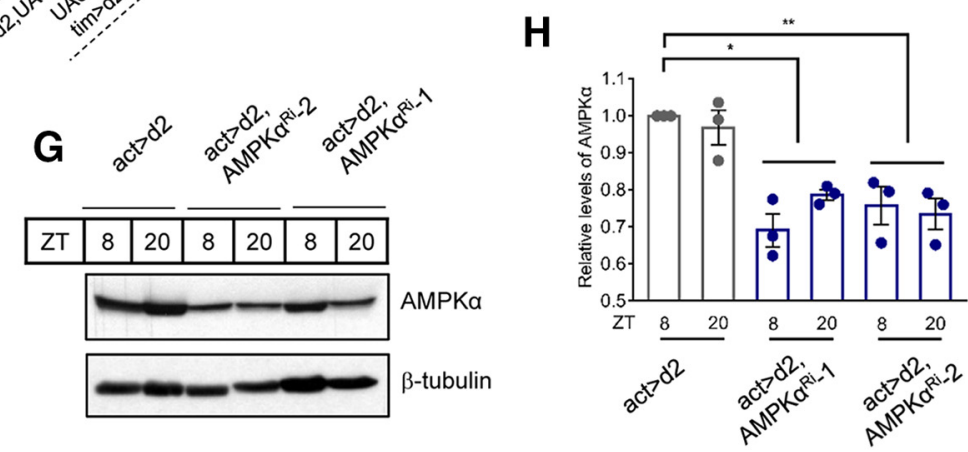

Figure 2. Circadian locomotor behaviors were altered in AMPK subunit knockdown flies. $A, B$, Daily activity profiles of young adult male flies on the last day of $L D$ (top) representing a given

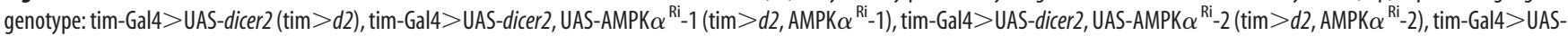

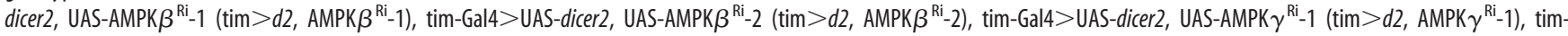

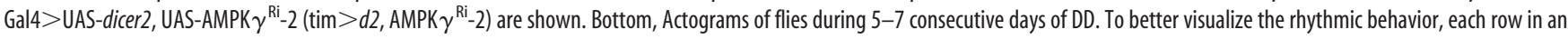
actogram was double-plotted. Light gray bars represent day-time activity. Dark gray bars represent subjective day-time activity. Black bars represent night-time and subjective night-time activity. Hatched horizontal bar represents subjective day. Black horizontal bar represents subjective night. ZT, Zeitgeber time. ZTO is light-on time. CT, Circadian time. CTO is subjective day start time. C, Total number of beam crossings of the single fly during the last day of LD was calculated and averaged for each given genotype of flies. Values indicate mean \pm SEM. Statistically significant differences in the total activity counts between UAS-AMPK ${ }^{\mathrm{Ri}}$ control flies and knockdown flies (Student's $t$ test): ${ }^{*} p<0.05 ;{ }^{* *} p<0.01$. D-F, Flies from the following strains were collected at ZT8 and ZT20:

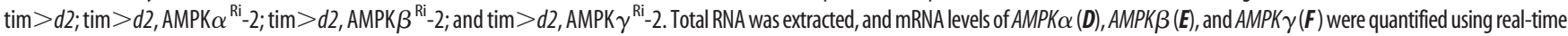
qRT-PCR. The mRNA levels in tim $>d 2$ at ZT8 were set to 1 , and the other values were normalized to these values. Values indicate mean \pm SEM of three independent (Figure legend continues.) 
A

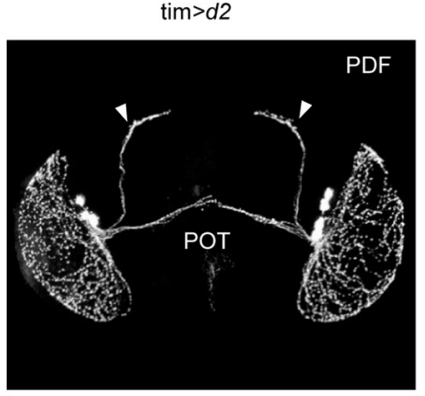

B

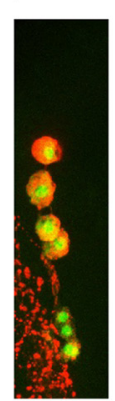

pdf $>$ nls-GFP

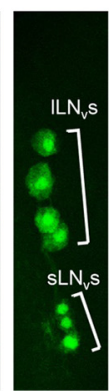

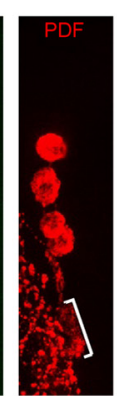

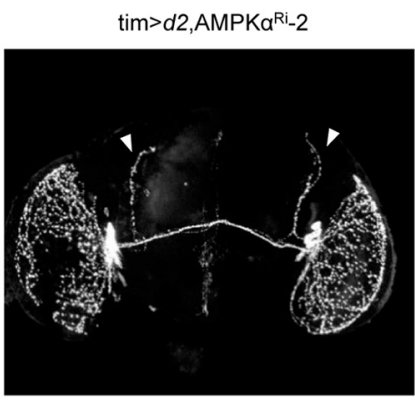

pdf $>$ nls-GFP, AMPK $\gamma^{\text {Ri-2 }}$
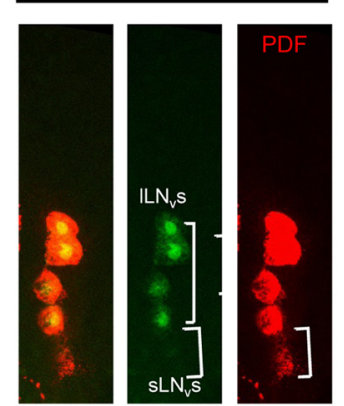

$\operatorname{tim}>d 2, A M P K \beta^{R i}-2$

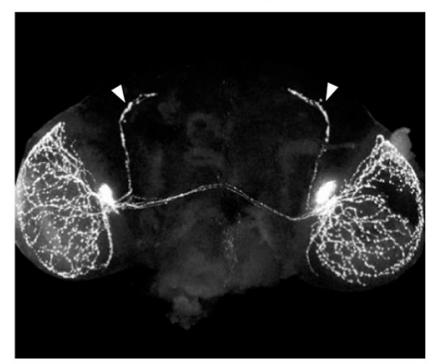

C

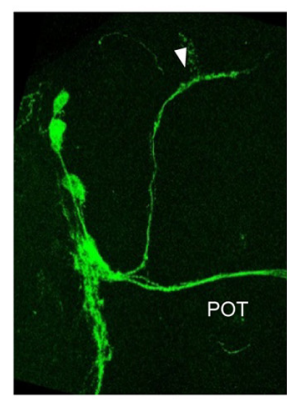

tim $>d 2$, AMPK $\gamma^{\text {Ri }}-2$

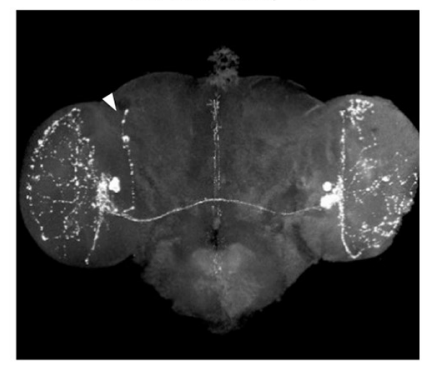

pdf $>m C D 8:: G F P, A M P K \gamma^{R i}-2$

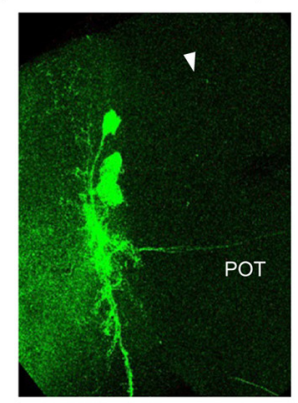

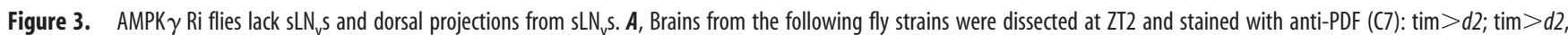

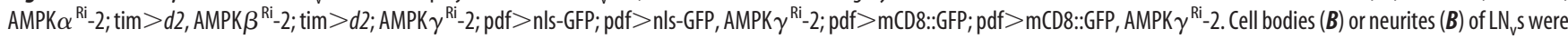
visualized by nls-GFP or mCD8::GFP expression under the control of pdf-Gal4. C, POT, Posterior optic tract. Arrowheads indicate dorsally projecting neurites from sL $\mathrm{N}_{v}$.

the control flies (Fig. 3B). In addition, dorsally projecting neurites that expressed mCD8::GFP were clearly visible in control flies but were absent in AMPK $\gamma$ Ri-2 flies (Fig. 3C). Thus, knockdown of AMPK $\gamma$ severely impaired the neuronal integrity of $s \mathrm{~N}_{\mathrm{v}}$ neurons but not $\mathrm{LN}_{\mathrm{v}}$ neurons. Previous reports have shown that the anticipation of light-on and light-off during LD is primarily controlled by $\operatorname{lLN}_{\mathrm{v}}$ neuronal activity (Cusumano et al., 2009; Sheeba et al., 2010). Further, it has been shown that $\mathrm{SLN}_{\mathrm{v}}$ neurons are required to synchronize the clock neuronal network to maintain the circadian rhythmicity under DD (Renn et al., 1999; Peng et al., 2003; Lin et al., 2004). In AMPK $\gamma$ Ri flies, quasi-normal daily locomotor activity during LD and complete loss of rhythmicity under DD could be attributed to intact $\operatorname{lLN}_{\mathrm{v}}$ neurons and impaired $\mathrm{sLN}_{\mathrm{v}}$ neurons.

The morphological defects observed in the $\mathrm{sLN}_{\mathrm{v}} \mathrm{s}$ of AMPK $\gamma$ Ri-2 flies led us to examine whether the circadian behavior phenotypes manifested by AMPK downregulation are caused by developmental effects. To test this, we used the TARGET system with tubulin-Gal80 ${ }^{\text {ts }}$ (McGuire et al., 2004). Flies were raised at the permissive temperature $\left(18^{\circ} \mathrm{C}\right)$ at which Gal $80^{\text {ts }}$ inhibited expression of UAS-AMPK ${ }^{\mathrm{Ri}}$. Then, locomotor behavior analysis was conducted at the permissive temperature and the restrictive temperature $\left(29^{\circ} \mathrm{C}\right)$. At $29^{\circ} \mathrm{C}, \mathrm{Gal} 80^{\text {ts }}$ does not function and Gal4 activates the expression of UAS-AMPK ${ }^{\mathrm{Ri}}$. In control flies, the

$\leftarrow$

(Figure legend continued.) experiments. Student's $t$ test: ${ }^{*} p<0.05 ;{ }^{* *} p<0.01 ;{ }^{* * *} p<$ $0.001{ }^{* * * *} p<0.0001$. G, Protein extracts were prepared from larvae of the indicated genotypes at ZT8 and ZT20 and were analyzed by immunoblotting with anti-AMPK $\alpha$ antibody. $\beta$-Tubulin served as the loading control. A representative image from three independent experiments is shown. $\boldsymbol{H}$, Quantification of AMPK $\alpha$ protein levels was performed with ImageJ. The AMPK $\alpha$ level in actin $>d 2$ at ZT 8 was set to 1 , and the other values were normalized to this value. Values indicate mean \pm SEM of three independent experiments. One-way ANOVA with Tukey's test: ${ }^{*} p<0.05 ;{ }^{* *} p<0.01 ;{ }^{* * *} p<0.001$. period was shortened by $\sim 0.6 \mathrm{~h}$ with enhanced rhythmicity at $29^{\circ} \mathrm{C}$ compared with the period and rhythmicity at $18^{\circ} \mathrm{C}$, although the period difference was not statistically significant (Table 1). The periods in AMPK $\alpha^{\mathrm{Ri}}-2, \mathrm{AMPK} \beta^{\mathrm{Ri}}-2$, and AMPK $\gamma^{\mathrm{Ri}}-2$ flies were $\sim 24 \mathrm{~h}$ at $18^{\circ} \mathrm{C}$. At $29^{\circ} \mathrm{C}$, the periods in $\mathrm{AMPK} \alpha \alpha^{\mathrm{Ri}}-2$ and $\mathrm{AMPK} \beta^{\mathrm{Ri}}-2$ flies were lengthened by $\sim 0.5 \mathrm{~h}$ and $\sim 1 \mathrm{~h}$, respectively, and rhythmicity was enhanced, whereas the period in AMPK $\gamma^{\mathrm{Ri}}-2$ flies was shortened by $\sim 0.8 \mathrm{~h}$ and rhythmicity was greatly reduced. The period alterations between $18^{\circ} \mathrm{C}$ and $29^{\circ} \mathrm{C}$ in $\mathrm{AMPK} \alpha^{\mathrm{Ri}}-2, \mathrm{AMPK} \beta^{\mathrm{Ri}}-2$, and $\mathrm{AMPK} \gamma^{\mathrm{Ri}}-2$ flies were statistically significant. Thus, behavioral analysis demonstrates that the depletion of AMPK $\alpha$ and AMPK $\beta$ subunits in adult flies in an acute manner appears to be sufficient to lengthen the period, and depletion of the AMPK $\gamma$ subunit sufficient to reduce rhythmicity. Compared with life-long knockdowns, we observed weaker effects on period and rhythmicity, probably because the duration of $\mathrm{AMPK}^{\mathrm{Ri}}$ expression was shorter; and thereby, the knockdown efficiency was lower. These data indicate that the circadian rhythm phenotypes manifested by downregulation of AMPK subunits are due to active requirements in clock cells and not to secondary effects on development.

Next, we performed PER immunostaining at ZT2 in control and AMPK subunit knockdown flies (Fig. 4A-D). PER-stained $s N_{v} s$ were missing in AMPK $\alpha$ Ri-2 and AMPK $\gamma$ Ri-2 flies, and the dorsally projecting neurites from the $s \mathrm{LN}_{\mathrm{v}} \mathrm{s}$ were absent in AMPK $\gamma$ Ri-2 flies and were fragmented in AMPK $\alpha$ Ri- 2 flies. On the other hand, $\mathrm{sLN}_{\mathrm{v}} \mathrm{s}$ and the dorsally projecting neurites from the $\mathrm{SLN}_{\mathrm{v}} \mathrm{s}$ were intact in the AMPK $\beta$ Ri-2 flies. PER levels were quantified in $\mathrm{lLN}_{\mathrm{v}} \mathrm{s}, \mathrm{LN}_{\mathrm{d}} \mathrm{s}$, and $\mathrm{DN}_{1} \mathrm{~s}$ (Fig. $4 E-G$ ). In all AMPK subunit knockdown flies, PER levels were reduced in $\operatorname{LN}_{\mathrm{v}} \mathrm{s}, \mathrm{LN}_{\mathrm{d}} \mathrm{s}$, and $\mathrm{DN}_{1} \mathrm{~s}$ compared with control flies, although some differences were not statistically significant. It appears that the morphological defects in the $\mathrm{sLN}_{\mathrm{v}} \mathrm{s}$ were most severe in the $\gamma$ knockdown, 

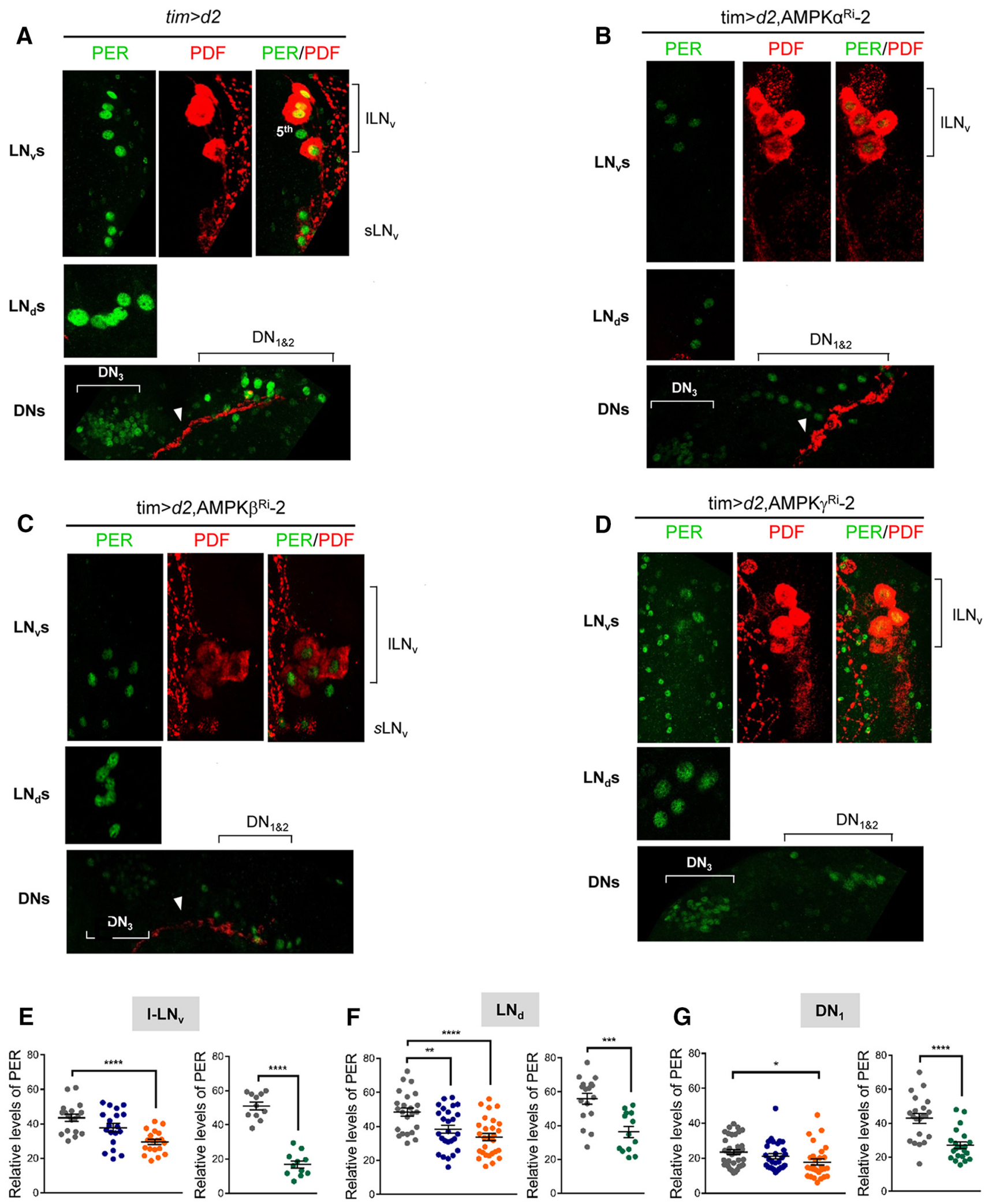

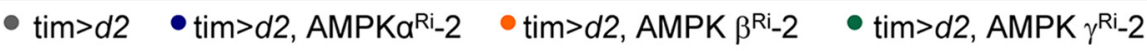

Figure 4. PER levels were reduced in pacemaker neurons of AMPK subunit knockdown flies. $A, B, C, D, B$ Bains from flies of the indicated genotypes were dissected at ZT2 and stained with anti-PER (Rb1,

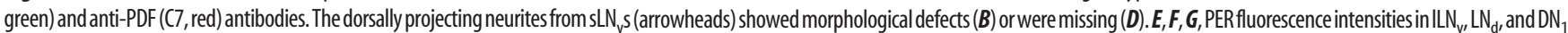
neurons were quantified. Values indicate mean \pm SEM $(n=11-35)$. One-way ANOVA with Tukey's test: ${ }^{*} p<0.05 ;{ }^{* *} p<0.01 ;{ }^{* * *} p<0.001$; $\left.{ }^{* * *} p<0.0001\right)$. 
A
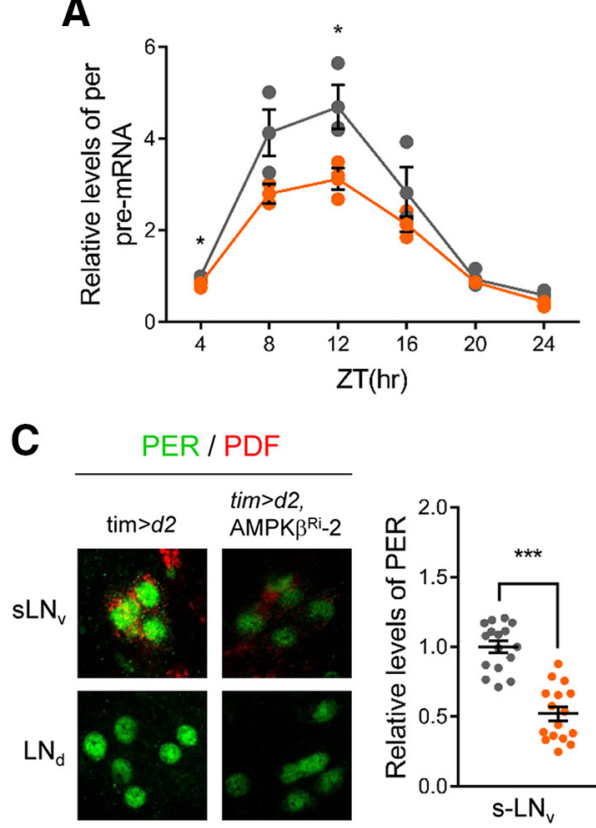

B

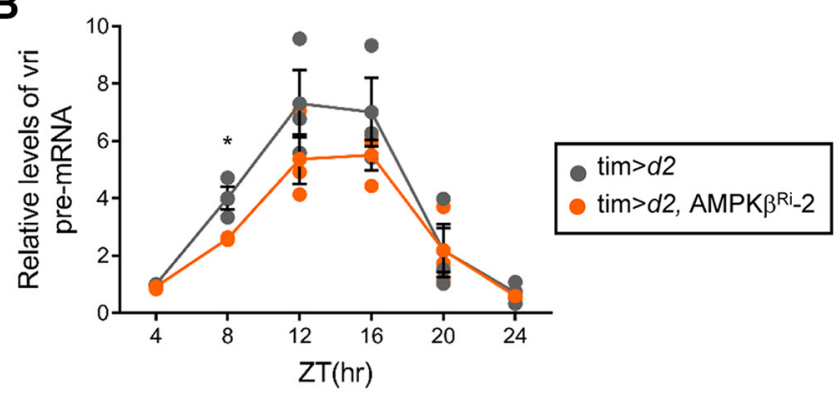

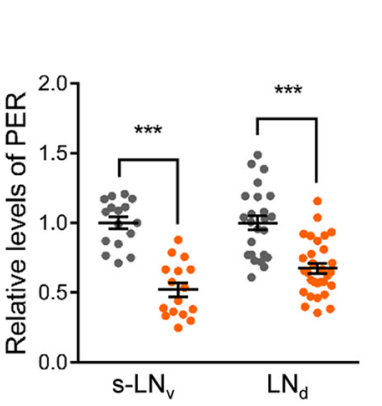
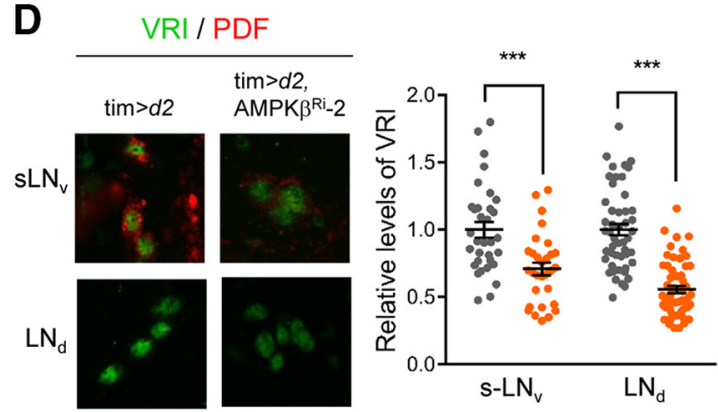

Figure 5. Transcription of CLK-target core clock gene is reduced in AMPK $\beta$ Ri flies. $A, B$, Flies of the indicated genotype were collected during LD and total RNA was isolated. Pre-mRNA levels of $\operatorname{per}(\boldsymbol{A})$ and $\operatorname{vri}(\boldsymbol{B})$ were quantified using real-time qRT-PCR. The pre-mRNA levels in $\operatorname{tim}>d 2$ at ZT4 were set to 1 , and the other values were normalized to these values. Values indicate mean $\pm S E M$ of three independent experiments. ${ }^{*} p<0.05$ (Student's $t$ test). C, D, Flies of the indicated genotypes were collected at ZT2 ( $C$ ) and ZT17 (D) during LD. The brains were stained with anti-PER (Rb1, green), anti-VRI (gp2, green), and anti-PDF (red) antibodies. Representative images of $s \mathrm{LN}_{\mathrm{v}} \mathrm{s}$ and $\mathrm{LN}_{\mathrm{d}} \mathrm{s}$ from two independent experiments are shown. PER and VRI fluorescence intensities were quantified. Values indicate mean $\pm \operatorname{SEM}(n=16-56) .{ }^{*} p<0.05$ (Student's $t$ test). ${ }^{* * *} p<0.001$ (Student's $t$ test).

then the $\alpha$ knockdown, and were the least severe in the $\beta$ knockdown; thus, the highly arrhythmic locomotor behaviors in the $\alpha$ and $\gamma$ Ri flies were likely caused by damaged $s \mathrm{LN}_{\mathrm{v}} \mathrm{s}$. Nonetheless, reduced PER levels in clock neurons were observed in all three AMPK subunit knockdown flies. Our data suggest that AMPK enzymatic activity is required to maintain the integrity and cellular oscillator function of $s \mathrm{~N}_{\mathrm{v}}$ neurons. Because $\mathrm{sLN}_{\mathrm{v}} \mathrm{s}$ are required for synchronizing circadian neural network for locomotor rhythm, we decided to use the AMPK $\beta$ knockdown flies containing intact $s \mathrm{LN}_{\mathrm{v}} \mathrm{s}$ to further examine the biochemical function of AMPK in the cellular oscillator.

\section{AMPK $\beta$ knockdown reduced CLK-driven transcription by reducing CLK levels}

Intriguingly, $\mathrm{sLN}_{\mathrm{v}} \mathrm{s}$ and dorsal projecting neurites are missing in Clk and $c y c$ mutants (Blau and Young, 1999; Park et al., 2000; Allada et al., 2003), phenocopying the situation when AMPK $\alpha$ or AMPK $\gamma$ was downregulated (Fig. 3). In addition, because AMPK interacted with CLK (Fig. 1), we reasoned that CLK/CYC activity may be affected in AMPK subunit knockdown flies. To examine whether CLK/CYC-mediated transcription was affected in AMPK $\beta$ subunit knockdown flies, we determined the pre-mRNA levels of per and $v r i$, which are core clock genes that are targeted by CLK/CYC (Fig. $5 A, B$ ). It has been established that pre-mRNA levels can be used as a read-out of transcription (Menet et al., 2010; Luo et al., 2012). Both per and vri pre-mRNA levels were reduced in $\mathrm{AMPK} \beta$ subunit knockdown flies, indicating that downregulation of AMPK $\beta$ reduced CLK/CYC-dependent transcription. Next, we performed immunostaining to examine PER and VRI protein levels at their peak time points: that is, ZT2, and ZT17, respectively, in $\mathrm{sLN}_{\mathrm{v}} \mathrm{s}$ and $\mathrm{LN}_{\mathrm{d}} \mathrm{s}$ (Fig. 5C,D). Consistent with the reduction in CLK/CYC-dependent transcription, we found that PER and VRI protein levels were reduced. The re- duced PER and VRI protein levels may have impeded the pace of the circadian clock in AMPK $\beta$ Ri-2 flies.

To determine whether AMPK directly phosphorylates CLK, we performed an in vitro kinase assay using purified AMPK holoenzyme and bacterially expressed GST-tagged CLK as the substrate. We found that AMPK phosphorylates CLK and that the phosphorylation was greatly enhanced by the addition of AMP (Fig. 6A). To assess how AMPK-mediated phosphorylation affects CLK, we examined daily CLK profiles. Hyperphosphorylated isoforms of CLK were observed in the early-day and late-night periods (ZT4, ZT20, and ZT23.8), and hypophosphorylated isoforms of CLK were observed during mid-day (ZT8 to ZT16) (Fig. 6B). A side-by-side comparison of CLK showed no significant differences in phosphorylation pattern in control and AMPK $\beta$ Ri- 2 flies. In addition, CLK protein levels in whole-head extracts from control and AMPK $\beta$ knockdown flies were similar throughout the day (Fig. $6 C$ ). Nevertheless, we reasoned that the effect of AMPK on CLK protein may be different in clock cells, and we tested this by immunostaining. Indeed, CLK levels were lower in $\mathrm{sLN}_{\mathrm{v}} \mathrm{s}$ and $\mathrm{LN}_{\mathrm{d}} \mathrm{s}$ in AMPK $\beta$ knockdown flies than in control flies (Fig. 6D). Moreover, both PER and CLK levels in $\mathrm{sLN}_{\mathrm{v}} \mathrm{s}$ were lower in flies in which AMPK $\beta$ was downregulated in an acute manner via the Gal80 ${ }^{\text {ts }}$ TARGET system than in control flies (Fig. 6E). Collectively, these observations indicate that AMPK-mediated phosphorylation stabilizes CLK in pacemaker neurons and enhances expression of CLK/CYC target genes.

\section{Expression of CLK rescued the arrhythmicity caused by the} AMPK $\boldsymbol{\beta}$ knockdown

If AMPK downregulation slowed the clock by affecting CLK levels, overexpression of CLK may rescue the circadian locomotor rhythm defect caused by AMPK $\beta$ knockdown. Because overexpression of $C l k$ in combination with the tim-GAL4 driver is de- 
A

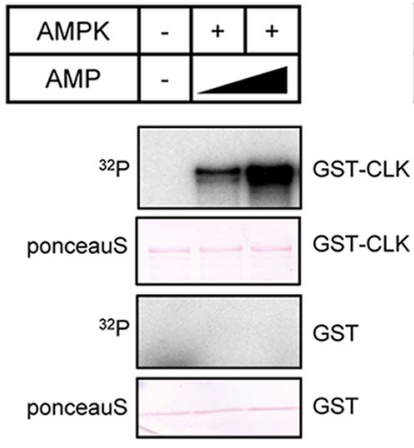

B

\begin{tabular}{|c|c|c|c|c|c|c|c|c|c|c|c|c|}
\hline ZT(h) & \multicolumn{2}{|c|}{4} & \multicolumn{2}{|c|}{8} & \multicolumn{2}{|c|}{11.8} & \multicolumn{2}{|c|}{16} & \multicolumn{2}{|c|}{20} & \multicolumn{2}{|c|}{23.8} \\
\hline flies & $\mathrm{Ct}$ & $\mathrm{Ri}$ & $\mathrm{Ct}$ & $\mathrm{Ri}$ & $\mathrm{Ct}$ & $\mathrm{Ri}$ & $\mathrm{Ct}$ & $\mathrm{Ri}$ & $\mathrm{Ct}$ & $\mathrm{Ri}$ & $\mathrm{C}$ & $\mathrm{Ri}$ \\
\hline
\end{tabular}
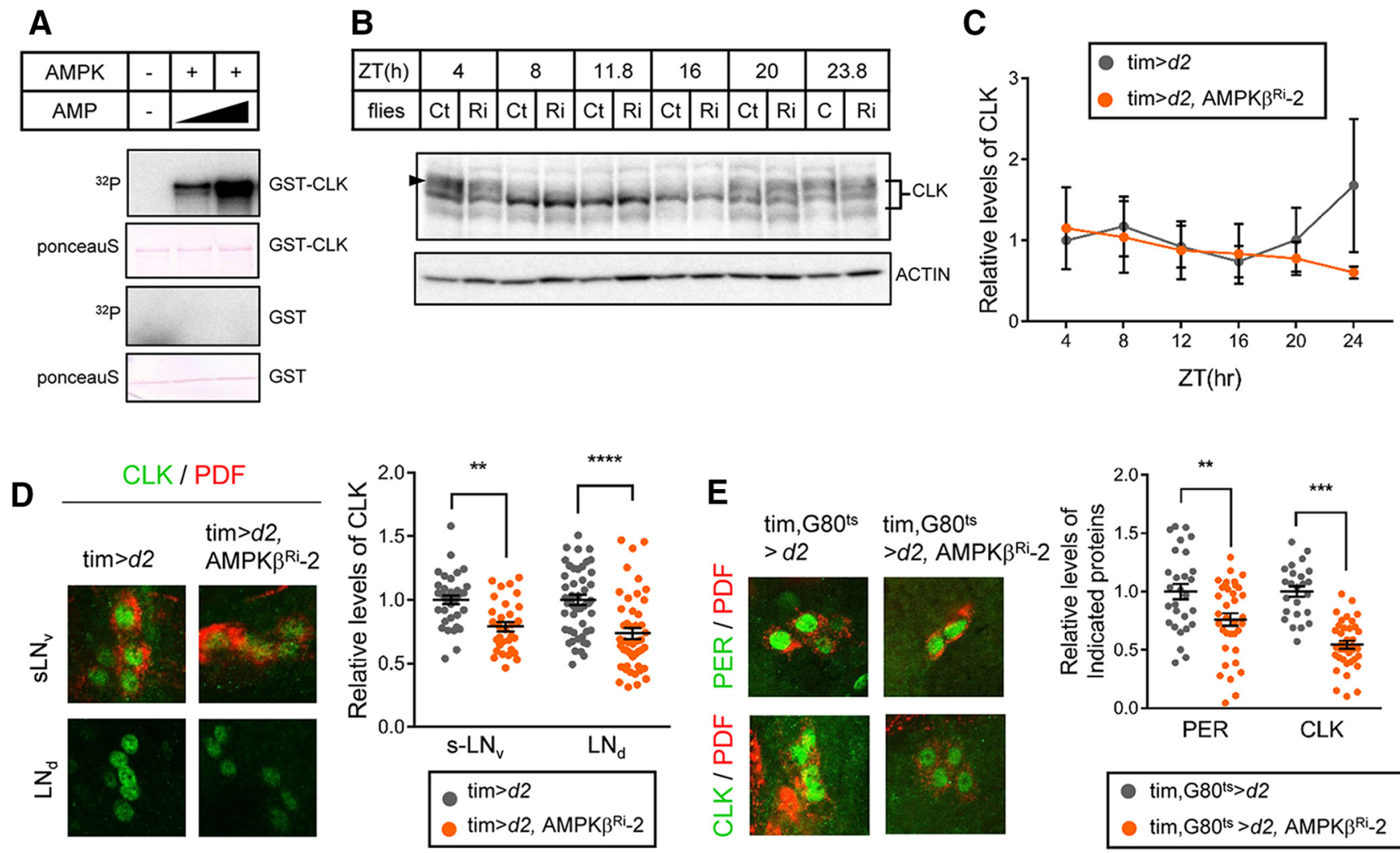

Figure 6. AMPK directly phosphorylated CLKin vitro and stabilized CLK in clock neurons. A, GST-CLK and GST purified from E. coli were incubated with AMPK holoenzyme and $\gamma^{-32}{ }^{32}$-ATP with increasing amounts of AMP. The samples were then subjected to SDS-PAGE, and the proteins were transferred to PVDF membranes. Following Ponceau $S$ staining, phosphorylation of GST-CLK and GST was visualized by autoradiography. $\boldsymbol{B}$, Protein extracts from the heads of control (Ct) and AMPK $\beta$ knockdown flies (Ri) were prepared at the indicated ZT and were analyzed by immunoblotting with anti-CLK (gp208) antibody. Actin served as the loading control. C, Relative levels of CLK were determined by measuring band intensities using ImageJ software. Values indicate mean \pm SEM $(n=3)$. D, Flies of the indicated genotypes were collected at ZT24 during LD, and the brains were stained with antibodies to CLK (gp50, green) and PDF (C7, red). Representative images of $s \mathrm{LN}_{v} s$ and $\mathrm{LN}_{\mathrm{d}} \mathrm{s}$ from two independent experiments are shown. CLK fluorescence intensities were quantified. Values indicate mean \pm SEM $(n=24-53)$. Statistically significant differences between control and AMPK $\beta$ Ri flies (Student's $t$ test): ${ }^{* *} p<0.01 ; *^{* *} p<0.001 ;{ }^{* * *} p<0.0001$. E, Flies of the indicated genotypes were collected at $Z T 2$ at $29^{\circ} \mathrm{C}$. The brains were costained with antibodies to CLK (gp50, green) and PDF (C7, red) or PER (Rb1, green) and PDF (C7, red). Representative images of sLN $\mathrm{N}_{y}$ are shown. CLK and PER fluorescence intensities were quantified. Values indicate mean \pm SEM $(n=24-37)$. Statistically significant differences between control and AMPK $\beta$ Ri flies (Student's $t$ test): ${ }^{* *} p<0.01 ;{ }^{* * *} p<0.001$.

velopmentally lethal (Zhao et al., 2003), we expressed UAS-Clk under control of the pdf-GAL4 driver. We confirmed overexpression of CLK by immunostaining $\operatorname{sLN}_{\mathrm{v}} \mathrm{s}$ (Fig. $7 A, B$ ). Knockdown of AMPK $\beta$ by the pdf-GAL4 driver in $\mathrm{LN}_{\mathrm{v}} \mathrm{s}$ was sufficient to cause long periods similar to the AMPK $\beta$ knockdown in the tim-expressing cells and to downregulate CLK levels in $\mathrm{sLN}_{\mathrm{v}} \mathrm{s}$ (Table 1; Fig. $7 D$ ). Overexpression of CLK suppressed the long-period phenotype of the AMPK $\beta$ knockdown and restored the $24 \mathrm{~h}$ period (Fig. $7 C$ ). These results indicate that the key mechanism underlying the circadian locomotor defect caused by AMPK $\beta$ knockdown in flies is downregulation of CLK levels.

\section{Discussion}

Daily rhythmic environmental changes entrain the circadian clock system of an organism to manifest the appropriate physiology and behavior on a circadian time-scale. In eukaryotes, oscillation of gene expression driven by circadian transcription factors is the basic framework for the cell-autonomous oscillator (for reviews, see Dunlap and Loros, 2004; Hardin, 2005; Takahashi, 2017). Thus, regulation of circadian transcription factors is fundamental to synchronizing the clock system with environmental changes. Here, we report that the AMPK $\gamma$ subunit copu- rified with CLK in Drosophila S2 cells and that AMPK activity is required for circadian locomotor rhythms because it enhances CLK-dependent transcriptional activation in the core clock neurons of Drosophila.

AMPK functions as a holoenzyme consisting of a catalytic $\alpha$ subunit and two regulatory subunits, $\beta$ and $\gamma$. In mammals, full activation of AMPK occurs upon phosphorylation of Thr172 (analogous to Thr184 in Drosophila) of the $\alpha$ subunit by upstream kinases, such as LKB and CAMKK2, which are activated by energy stress and an increase in the intracellular $\mathrm{Ca}^{2+}$ level, respectively (for review, see Ross et al., 2016; Garcia and Shaw, 2017). AMP binding to the $\gamma$ subunit prolongs Thr172 phosphorylation of the $\alpha$ subunit, thereby enhancing AMPK activity (Xiao et al., 2011; Gowans et al., 2013). The $\beta$ subunit plays a stochiometric and stabilizing role in the trimeric complex by binding the $\alpha$ and $\gamma$ subunits in a 1:1:1 ratio (Woods et al., 1996). Binding of a novel AMPK-activating compound to the AMPK $\beta$ subunit protects the AMPK $\alpha$ subunit Thr172 from dephosphorylation, indicating that the AMPK $\beta$ subunit also regulates the phosphorylation status of AMPK $\alpha$ (Göransson et al., 2007; Sanders et al., 2007). Consistently, mutant mice lacking AMPK $\beta 1$ exhibit severe brain abnormalities caused by reduced AMPK activity (Dasgupta and Milbrandt, 2009). Consequently, in 
A

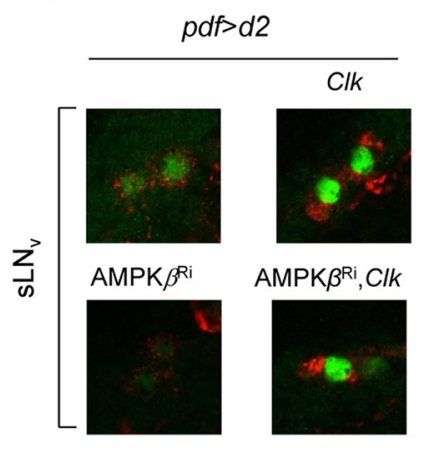

CLK I PDF

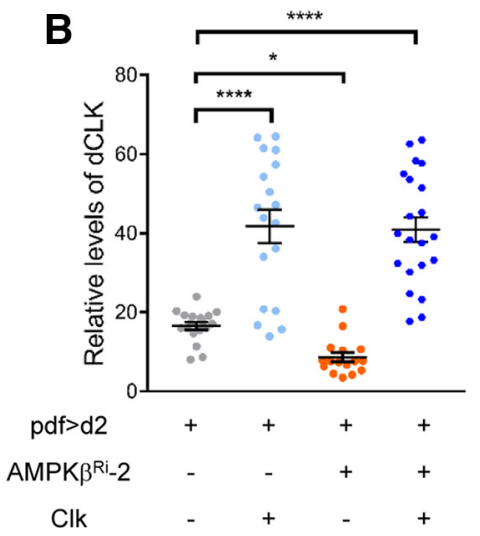

D
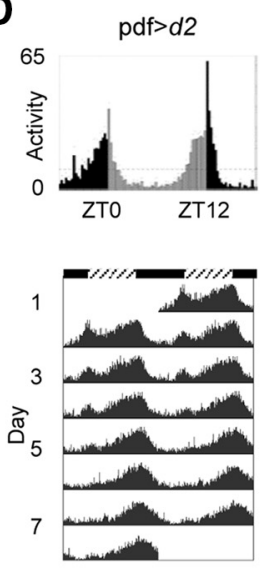
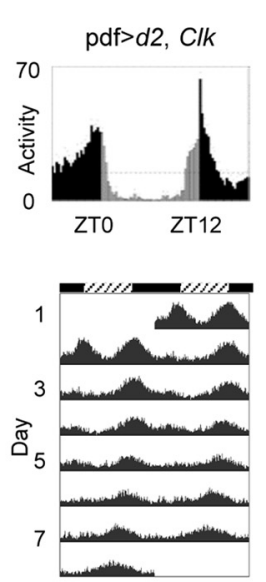

C

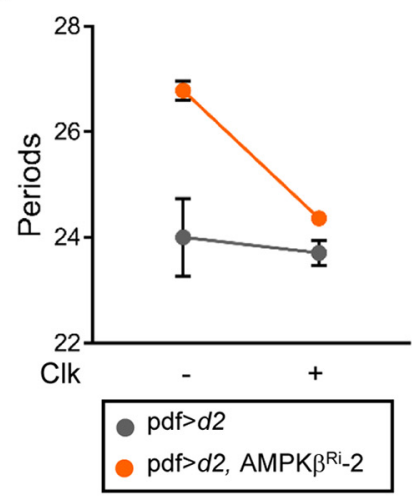

Figure 7. Expression of Clk rescued the long period and arrhythmicity induced by AMPK $\beta$ knockdown. $A, B$, Flies of the indicated genotypes were collected at ZT2 during LD. The brains were stained with anti-CLK (gp50, green) and anti-PDF (red) antibodies. Representative images of $s L \mathrm{~N}_{v} s$ are shown. CLK fluorescence intensities were quantified. Values indicate mean \pm

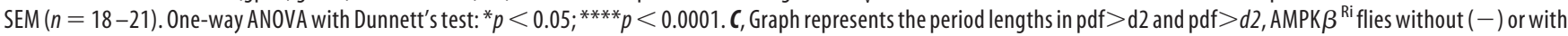
$(+)$ Clk overexpression by pdf-Gal4 driver. Values indicate mean \pm SEM. Two-way ANOVA showed significant differences in the AMPK $\beta$ knockdown $\left(F_{(1,84)}=26.23, p=1.83 \times 10^{-6}\right)$, Clk overexpression $\left(F_{(1,84)}=120.03, p=5.40 \times 10^{-18}\right)$, and interaction $\left(F_{(1,84)}=37.20, p=2.95 \times 10^{-8}\right)$. D. Daily activity profiles of young adult male flies of the indicated genotypes on the last day of LD (top) and on the first day of DD (middle) are shown. Bottom, Actograms of flies during 7 consecutive days of DD. To better visualize the rhythmic behavior, each row in an actogram was double-plotted. Light gray bars represent day-time activity. Dark gray bars represent subjective day-time activity. Black bars represent night-time and subjective night-time activity. Hatched horizontal bar represents subjective day. Black horizontal bar represents subjective night. ZT, Zeitgeber time. ZTO is light-on time. CT, Circadian time. CTO is subjective day start time.

this study, we reasoned that downregulation of expression of each AMPK subunit would reduce the activity of the AMPK holoenzyme. Our results showed that knockdown of each AMPK subunit produced circadian locomotor rhythm defects, supporting the notion that AMPK activity is required for the circadian rhythm in Drosophila.

In mammals, AMPK affects the core clock by regulating circadian repressor proteins. AMPK phosphorylates mCRY1 and mCRY2, which stimulates their interaction with F-box/LRRrepeat protein 3 (FBXL3), an E3 ligase, leading to degradation of CRY (Lamia et al., 2009). AMPK has also been shown to phosphorylate serine 389 of CKI $\varepsilon$, which enhances CKI $\varepsilon$ enzymatic activity and degradation of mPER2 (Um et al., 2011). Collectively, in mammals, AMPK destabilizes circadian repressor proteins and induces derepression of CLK/BMAL1-dependent transcription. The role of AMPK in regulating expression of clock genes was reported in skeletal muscle cells following treatment with 5-aminoimidazole-4-carboxamide ribonucleotide, a stimulator of AMPK activity, albeit the detailed molecular mechanism was not provided (Vieira et al., 2008).

In this study, we propose that, in Drosophila, AMPK regulates the core clock by increasing the activity and level of CLK. This proposition is based on several observations. First, if AMPK phosphorylates and destabilizes CRY as in mammals, then downregulation of AMPK subunits would result in increased CRY levels in Drosophila. However, previous studies have shown that overexpression of cry under the control of a tim-GAL4 or a $p d f$ GAL4 driver did not affect behavioral rhythms in Drosophila (Dissel et al., 2004; Collins et al., 2006). Thus, AMPK does not appear to affect the core clock by regulating CRY stability in Drosophila. Second, per and vri pre-mRNA levels were lower in AMPK $\beta$ knockdown flies than in control flies (Fig. 5), indicating that CLK/CYC-dependent transcription requires AMPK activity (Menet et al., 2010; Luo et al., 2012; Andreazza et al., 2015). We also showed that PER and VRI protein levels were lower in pacemaker neurons of AMPK $\beta$ knockdown flies than in the control flies, which is consistent with our pre-mRNA results. Furthermore, rescue of locomotor behavior in AMPK $\beta$ knockdown flies by $\mathrm{LN}_{\mathrm{v}}$-specific CLK expression strongly supports the notion that compromised CLK/CYC transcription underlies the circadian rhythm defect that occurs when AMPK is downregulated. Third, we showed that purified CLK protein is directly phosphorylated by AMPK in an AMP-dependent manner using the in vitro kinase assay (Fig. 6A). To identify the AMPK phosphorylation site(s) on 
CLK, we searched candidate phosphosites using Scansite 4.0 (https://scansite4.mit.edu/4.0/\#scanProtein). We mutated 10 Ser/Thr residues in CLK to alanine and used the in vitro kinase assay to evaluate phosphorylation of these CLK mutants. We did not observe any significant differences in the phosphorylation of these mutants compared with WT CLK (data not shown), perhaps because the predicted phosphosites were not the actual phosphosites or because alteration of a single residue is not enough to affect the degree of phosphorylation in vitro. Further studies are needed to determine the AMPK phosphorylation site(s) in CLK. Finally, the impaired integrity of the $\mathrm{sLN}_{\mathrm{v}} \mathrm{s}$ and the dorsal projections observed in AMPK $\alpha$ and AMPK $\gamma$ Ri flies matched the phenotype of flies with reduced CLK and CYC activities (Fig. 3; and see below). Thus, we conclude that AMPK regulates the core clock in Drosophila by activating CLK, a positive component of the clock. It is intriguing to note that, while the target of AMPK action is different in Drosophila and mammals (positive element vs negative element, respectively), the outcome is in the same direction (i.e., the enhancement of circadian transcription). Nonetheless, there is also possibility that other circadian clock protein(s) are affected by AMPK and regulate circadian rhythm.

Downregulation of the AMPK $\alpha$ or the AMPK $\gamma$ subunit in tim-expressing cells led to arrhythmicity accompanied with the disruption of $s \mathrm{LN}_{\mathrm{v}}$ cell bodies and dorsally projecting neurites. It has been shown that $s \mathrm{LN}_{\mathrm{v}} \mathrm{s}$ and dorsally projecting neurites are absent when CLK and CYC activities are compromised in $C l k^{\mathrm{Jrk}}$, $C l k^{\text {ar }}, C l k^{\text {out }}$, and $c y c^{0}$ mutants (Blau and Young, 1999; Park et al., 2000; Allada et al., 2003). In addition, it has recently been shown that overexpression of the dominant negative form of microtubule star, which encodes a catalytic subunit of PP2A, in timexpressing cells induces morphological defects in $\mathrm{sLN}_{\mathrm{v}} \mathrm{s}$ (Andreazza et al., 2015). Although the authors of the study did not discuss the possible underlying mechanism, because PP2A controls the phosphorylation status of CLK, which is critical for CLK stability and activity, dysregulation of CLK may underlie the defects in the $\mathrm{sLN}_{\mathrm{v}} \mathrm{s}$ in the microtubule star mutant (Kim and Edery, 2006; Andreazza et al., 2015). Furthermore, VRI, the downstream target of CLK/CYC, controls the accumulation of the neuropeptide PDF in a post-transcriptional manner (Blau and Young, 1999; Gunawardhana and Hardin, 2017). Thus, dysregulation of CLK/CYC activity may underlie the impaired integrity of $s L N_{v} s$ in AMPK $\alpha$ and AMPK $\gamma$ Ri flies, which is consistent with the observation that CLK is required for $\mathrm{sLN}_{\mathrm{v}}$ development and integrity (Lerner et al., 2015). Interestingly, transcriptome analysis revealed that $C l k$ expression is reduced in human epileptogenic tissue, and a reduction in Clk expression contributes to malformation of the dendritic spine. These data suggest that CLK transcriptional activity is required for neuronal structural integrity (Li et al., 2017).

ATP levels and $\mathrm{Ca}^{2+}$ levels, which are upstream signals of AMPK, have been shown to display daily rhythms in several tissues and organisms (Yamazaki et al., 1994; Ikeda et al., 2003; Enoki et al., 2012; Fustin et al., 2012; Goya et al., 2016). AMPK activity has also been shown to exhibit a daily rhythm in the hypothalamus, retina, liver, and fibroblasts in a cell-autonomous manner (Um et al., 2011; Barnea et al., 2012; Huang et al., 2015). The differences in the active phases and regulatory mechanisms in the tissues and cells suggest that daily AMPK rhythms may be controlled in a tissue- and cell-specific manner. We also sought to explore whether AMPK activity might show daily rhythm, coupled with regulation of CLK levels in Drosophila. Nonetheless, the
mRNA levels of the AMPK $\alpha, \beta$, and $\gamma$ subunits did not show diurnal oscillations (Fig. 2D-F, compare ZT8 and ZT20). Because the available anti-AMPK $\alpha$ antibody does not work in the immunostaining assay, Western blot analysis of whole fly head extracts was performed; no rhythms in daily AMPK $\alpha$ protein levels were observed (data not shown). However, the possibility that AMPK $\alpha$ levels oscillate in a cell-specific manner cannot be ruled out. Regarding AMPK activity, a widely used AMPK $\alpha$ Thr172 phosphospecific antibody could not reliably determine the phosphorylation status of Drosophila AMPK $\alpha$ Thr184 (data not shown). Thus, currently, it is not elucidated whether the daily rhythmic AMPK activity and/or levels are coupled to regulate rhythmic CLK/CYC-dependent transcription in a daily basis in Drosophila. Nevertheless, pronounced acute metabolic stress or an increase in the intracellular $\mathrm{Ca}^{2+}$ level can induce AMPK activation and enhance CLK/CYC-dependent transcription to reset the phase.

In Drosophila, the crosstalk mechanism between nutrients, energy metabolism, and the core clock has been elucidated. The nutrient signal regulator AKT and the TOR-S6K pathway determine the circadian period by regulating shaggy/glycogen synthase kinase $3 \beta$, thereby regulating nuclear entry of TIM (Zheng and Sehgal, 2010). O-GlcNAc modification of PER, which may be under the control of cellular glucose metabolism, sets the circadian period by regulating nuclear entry of PER (Kim et al., 2012; Kaasik et al., 2013). In this study, we showed that AMPK, which is sensitive to the AMP/ATP ratio and the internal $\mathrm{Ca}^{2+}$ concentration, regulates the circadian rhythm by regulating the level and activity of CLK.

\section{References}

Allada R, White NE, So WV, Hall JC, Rosbash M (1998) A mutant Drosophila homolog of mammalian clock disrupts circadian rhythms and transcription of period and timeless. Cell 93:791-804.

Allada R, Kadener S, Nandakumar N, Rosbash M (2003) A recessive mutant of Drosophila clock reveals a role in circadian rhythm amplitude. EMBO J 22:3367-3375.

Andreazza S, Bouleau S, Martin B, Lamouroux A, Ponien P, Papin C, Chélot E, Jacquet E, Rouyer F (2015) Daytime CLOCK dephosphorylation is controlled by STRIPAK complexes in Drosophila. Cell Rep 11:1266-1279.

Barnea M, Haviv L, Gutman R, Chapnik N, Madar Z, Froy O (2012) Metformin affects the circadian clock and metabolic rhythms in a tissuespecific manner. Biochim Biophys Acta 1822:1796-1806.

Blau J, Young MW (1999) Cycling vrille expression is required for a functional Drosophila clock. Cell 99:661-671.

Brand AH, Perrimon N (1993) Targeted gene expression as a means of altering cell fates and generating dominant phenotypes. Development 118: 401-415.

Collins B, Mazzoni EO, Stanewsky R, Blau J (2006) Drosophila CRYPTOCHROME is a circadian transcriptional repressor. Curr Biol 16:441-449.

Cusumano P, Klarsfeld A, Chélot E, Picot M, Richier B, Rouyer F (2009) PDF-modulated visual inputs and cryptochrome define diurnal behavior in Drosophila. Nat Neurosci 12:1431-1437.

Cyran SA, Yiannoulos G, Buchsbaum AM, Saez L, Young MW, Blau J (2005) The double-time protein kinase regulates the subcellular localization of the Drosophila clock protein period. J Neurosci 25:5430-5437.

Dasgupta B, Milbrandt J (2009) AMP-activated protein kinase phosphorylates retinoblastoma protein to control mammalian brain development. Dev Cell 16:256-270.

Dissel S, Codd V, Fedic R, Garner KJ, Costa R, Kyriacou CP, Rosato E (2004) A constitutively active cryptochrome in Drosophila melanogaster. Nat Neurosci 7:834-840.

Dunlap JC, Loros JJ (2004) The neurospora circadian system. J Biol Rhythms 19:414-424.

Enoki R, Ono D, Hasan MT, Honma S, Honma K (2012) Single-cell resolution fluorescence imaging of circadian rhythms detected with a Nipkow spinning disk confocal system. J Neurosci Methods 207:72-79.

Fustin JM, Doi M, Yamada H, Komatsu R, Shimba S, Okamura H (2012) 
Rhythmic nucleotide synthesis in the liver: temporal segregation of metabolites. Cell Rep 1:341-349.

Garcia D, Shaw RJ (2017) AMPK: mechanisms of cellular energy sensing and restoration of metabolic balance. Mol Cell 66:789-800.

Glossop NR, Houl JH, Zheng H, Ng FS, Dudek SM, Hardin PE (2003) VRILLE feeds back to control circadian transcription of clock in the Drosophila circadian oscillator. Neuron 37:249-261.

Göransson O, McBride A, Hawley SA, Ross FA, Shpiro N, Foretz M, Viollet B, Hardie DG, Sakamoto K (2007) Mechanism of action of A-769662, a valuable tool for activation of AMP-activated protein kinase. J Biol Chem 282:32549-32560.

Gowans GJ, Hawley SA, Ross FA, Hardie DG (2013) AMP is a true physiological regulator of AMP-activated protein kinase by both allosteric activation and enhancing net phosphorylation. Cell Metab 18:556-566.

Goya ME, Romanowski A, Caldart CS, Bénard CY, Golombek DA (2016) Circadian rhythms identified in Caenorhabditis elegans by in vivo longterm monitoring of a bioluminescent reporter. Proc Natl Acad Sci U S A 113:E7837-E7845.

Gunawardhana KL, Hardin PE (2017) VRILLE controls PDF neuropeptide accumulation and arborization rhythms in small ventrolateral neurons to drive rhythmic behavior in Drosophila. Curr Biol 27:3442-3453.e4.

Hardin PE (2005) The circadian timekeeping system of Drosophila. Curr Biol 15:R714-R722.

Hardin PE (2011) Molecular genetic analysis of circadian timekeeping in Drosophila. Adv Genet 74:141-173.

Helfrich-Förster C (1998) Robust circadian rhythmicity of Drosophila melanogaster requires the presence of lateral neurons: a brain-behavioral study of disconnected mutants. J Comp Physiol A 182:435-453.

Helfrich-Förster C (2005) Neurobiology of the fruit fly's circadian clock. Genes Brain Behav 4:65-76.

Helfrich-Förster C, Shafer OT, Wülbeck C, Grieshaber E, Rieger D, Taghert P (2007) Development and morphology of the clock-gene-expressing lateral neurons of Drosophila melanogaster. J Comp Neurol 500:47-70.

Houl JH, Yu W, Dudek SM, Hardin PE (2006) Drosophila CLOCK is constitutively expressed in circadian oscillator and non-oscillator cells. J Biol Rhythms 21:93-103.

Huang CC, Shi L, Lin CH, Kim AJ, Ko ML, Ko GY (2015) A new role for AMP-activated protein kinase in the circadian regulation of L-type voltage-gated calcium channels in late-stage embryonic retinal photoreceptors. J Neurochem 135:727-741.

Ikeda M, Sugiyama T, Wallace CS, Gompf HS, Yoshioka T, Miyawaki A, Allen CN (2003) Circadian dynamics of cytosolic and nuclear $\mathrm{Ca}^{2+}$ in single suprachiasmatic nucleus neurons. Neuron 38:253-263.

Kaasik K, Kivimäe S, Allen JJ, Chalkley RJ, Huang Y, Baer K, Kissel H, Burlingame AL, Shokat KM, Ptacek LJ, Fu YH (2013) Glucose sensor O-GlcNAcylation coordinates with phosphorylation to regulate circadian clock. Cell Metab 17:291-302.

Kadener S, Menet JS, Schoer R, Rosbash M (2008) Circadian transcription contributes to core period determination in Drosophila. PLoS Biol 6:e119.

Kim EY, Edery I (2006) Balance between DBT/CKIepsilon kinase and protein phosphatase activities regulate phosphorylation and stability of Drosophila CLOCK protein. Proc Natl Acad Sci U S A 103:6178-6183.

Kim EY, Jeong EH, Park S, Jeong HJ, Edery I, Cho JW (2012) A role for O-GlcNAcylation in setting circadian clock speed. Genes Dev 26:490502.

Kusch T, Florens L, Macdonald WH, Swanson SK, Glaser RL, Yates JR 3rd, Abmayr SM, Washburn MP, Workman JL (2004) Acetylation by Tip60 is required for selective histone variant exchange at DNA lesions. Science 306:2084-2087.

Lamia KA, Sachdeva UM, DiTacchio L, Williams EC, Alvarez JG, Egan DF, Vasquez DS, Juguilon H, Panda S, Shaw RJ, Thompson CB, Evans RM (2009) AMPK regulates the circadian clock by cryptochrome phosphorylation and degradation. Science 326:437-440.

Lee C, Bae K, Edery I (1999) PER and TIM inhibit the DNA binding activity of a Drosophila CLOCK-CYC/dBMAL1 heterodimer without disrupting formation of the heterodimer: a basis for circadian transcription. Mol Cell Biol 19:5316-5325.

Lee E, Jeong EH, Jeong HJ, Yildirim E, Vanselow JT, Ng F, Liu Y, Mahesh G, Kramer A, Hardin PE, Edery I, Kim EY (2014) Phosphorylation of a central clock transcription factor is required for thermal but not photic entrainment. PLoS Genet 10:e1004545.
Lee E, Cho E, Kang DH, Jeong EH, Chen Z, Yoo SH, Kim EY (2016) Pacemaker-neuron-dependent disturbance of the molecular clockwork by a Drosophila CLOCK mutant homologous to the mouse clock mutation. Proc Natl Acad Sci U S A 113:E4904-E4913.

Lerner I, Bartok O, Wolfson V, Menet JS, Weissbein U, Afik S, Haimovich D, Gafni C, Friedman N, Rosbash M, Kadener S (2015) Clk posttranscriptional control denoises circadian transcription both temporally and spatially. Nat Commun 6:7056.

Li P, Fu X, Smith NA, Ziobro J, Curiel J, Tenga MJ, Martin B, Freedman S, Cea-Del Rio CA, Oboti L, Tsuchida TN, Oluigbo C, Yaun A, Magge SN, O’Neill B, Kao A, Zelleke TG, Depositario-Cabacar DT, Ghimbovschi S, Knoblach S, et al. (2017) Loss of CLOCK results in dysfunction of brain circuits underlying focal epilepsy. Neuron 96:387-401.e6.

Lin Y, Stormo GD, Taghert PH (2004) The neuropeptide pigmentdispersing factor coordinates pacemaker interactions in the Drosophila circadian system. J Neurosci 24:7951-7957.

Luo W, Li Y, Tang CH, Abruzzi KC, Rodriguez J, Pescatore S, Rosbash M (2012) CLOCK deubiquitylation by USP8 inhibits CLK/CYC transcription in Drosophila. Genes Dev 26:2536-2549.

McGuire SE, Mao Z, Davis RL (2004) Spatiotemporal gene expression targeting with the TARGET and gene-switch systems in Drosophila. Sci STKE 2004:pl6.

Menet JS, Abruzzi KC, Desrochers J, Rodriguez J, Rosbash M (2010) Dynamic PER repression mechanisms in the Drosophila circadian clock: from on-DNA to off-DNA. Genes Dev 24:358-367.

Nagarkar-Jaiswal S, Manivannan SN, Zuo Z, Bellen HJ (2017) A cell cycleindependent, conditional gene inactivation strategy for differentially tagging wild-type and mutant cells. Elife 6:e26420.

Nitabach MN, Blau J, Holmes TC (2002) Electrical silencing of Drosophila pacemaker neurons stops the free-running circadian clock. Cell 109:485495.

Pan DA, Hardie DG (2002) A homologue of AMP-activated protein kinase in Drosophila melanogaster is sensitive to AMP and is activated by ATP depletion. Biochem J 367:179-186.

Park JH, Helfrich-Förster C, Lee G, Liu L, Rosbash M, Hall JC (2000) Differential regulation of circadian pacemaker output by separate clock genes in Drosophila. Proc Natl Acad Sci U S A 97:3608-3613.

Partch CL, Green CB, Takahashi JS (2014) Molecular architecture of the mammalian circadian clock. Trends Cell Biol 24:90-99.

Peng Y, Stoleru D, Levine JD, Hall JC, Rosbash M (2003) Drosophila freerunning rhythms require intercellular communication. PLoS Biol 1:E13.

Renn SC, Park JH, Rosbash M, Hall JC, Taghert PH (1999) A pdf neuropeptide gene mutation and ablation of PDF neurons each cause severe abnormalities of behavioral circadian rhythms in Drosophila. Cell 99:791-802.

Ross FA, MacKintosh C, Hardie DG (2016) AMP-activated protein kinase: a cellular energy sensor that comes in 12 flavours. FEBS J 283:2987-3001.

Rutila JE, Suri V, Le M, So WV, Rosbash M, Hall JC (1998) CYCLE is a second bHLH-PAS clock protein essential for circadian rhythmicity and transcription of Drosophila period and timeless. Cell 93:805-814.

Saez L, Young MW (1996) Regulation of nuclear entry of the Drosophila clock proteins period and timeless. Neuron 17:911-920.

Sanders MJ, Ali ZS, Hegarty BD, Heath R, Snowden MA, Carling D (2007) Defining the mechanism of activation of AMP-activated protein kinase by the small molecule A-769662, a member of the thienopyridone family. J Biol Chem 282:32539-32548.

Schmid B, Helfrich-Förster C, Yoshii T (2011) A new ImageJ plug-in "ActogramJ” for chronobiological analyses. J Biol Rhythms 26:464-467.

Sheeba V, Fogle KJ, Holmes TC (2010) Persistence of morning anticipation behavior and high amplitude morning startle response following functional loss of small ventral lateral neurons in Drosophila. PLoS One 5:e11628.

Szabó A, Papin C, Zorn D, Ponien P, Weber F, Raabe T, Rouyer F (2013) The CK2 kinase stabilizes CLOCK and represses its activity in the Drosophila circadian oscillator. PLoS Biol 11:e1001645.

Takahashi JS (2017) Transcriptional architecture of the mammalian circadian clock. Nat Rev Genet 18:164-179.

Um JH, Pendergast JS, Springer DA, Foretz M, Viollet B, Brown A, Kim MK, Yamazaki S, Chung JH (2011) AMPK regulates circadian rhythms in a tissue- and isoform-specific manner. PLoS One 6:e18450.

Vieira E, Nilsson EC, Nerstedt A, Ormestad M, Long YC, Garcia-Roves PM, 
Zierath JR, Mahlapuu M (2008) Relationship between AMPK and the transcriptional balance of clock-related genes in skeletal muscle. Am J Physiol Endocrinol Metab 295:E1032-E1037.

Woods A, Cheung PC, Smith FC, Davison MD, Scott J, Beri RK, Carling D (1996) Characterization of AMP-activated protein kinase beta and gamma subunits: assembly of the heterotrimeric complex in vitro. J Biol Chem 271:10282-10290.

Xiao B, Sanders MJ, Underwood E, Heath R, Mayer FV, Carmena D, Jing C, Walker PA, Eccleston JF, Haire LF, Saiu P, Howell SA, Aasland R, Martin SR, Carling D, Gamblin SJ (2011) Structure of mammalian AMPK and its regulation by ADP. Nature 472:230-233.

Yamazaki S, Ishida Y, Inouye S (1994) Circadian rhythms of adenosine triphosphate contents in the suprachiasmatic nucleus, anterior hypotha- lamic area and caudate putamen of the rat: negative correlation with electrical activity. Brain Res 664:237-240.

Yu W, Zheng H, Houl JH, Dauwalder B, Hardin PE (2006) PER-dependent rhythms in CLK phosphorylation and E-box binding regulate circadian transcription. Genes Dev 20:723-733.

Yu W, Houl JH, Hardin PE (2011) NEMO kinase contributes to core period determination by slowing the pace of the Drosophila circadian oscillator. Curr Biol 21:756-761.

Zhao J, Kilman VL, Keegan KP, Peng Y, Emery P, Rosbash M, Allada R (2003) Drosophila clock can generate ectopic circadian clocks. Cell 113:755-766.

Zheng X, Sehgal A (2010) AKT and TOR signaling set the pace of the circadian pacemaker. Curr Biol 20:1203-1208. 